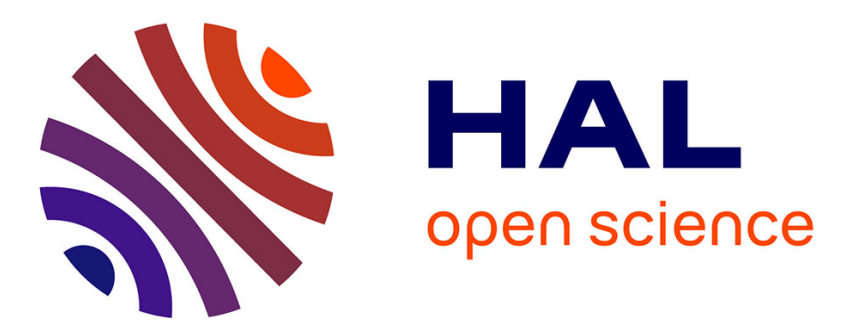

\title{
A belief function theory based approach to combining different representation of uncertainty in prognostics
}

Piero Baraldi, Francesca Mangili, Enrico Zio

\section{To cite this version:}

Piero Baraldi, Francesca Mangili, Enrico Zio. A belief function theory based approach to combining different representation of uncertainty in prognostics. Information Sciences, 2015, 303, pp.134-149. 10.1016/j.ins.2014.12.051 . hal-01265649

\section{HAL Id: hal-01265649 \\ https://hal.science/hal-01265649}

Submitted on 1 Feb 2016

HAL is a multi-disciplinary open access archive for the deposit and dissemination of scientific research documents, whether they are published or not. The documents may come from teaching and research institutions in France or abroad, or from public or private research centers.
L'archive ouverte pluridisciplinaire HAL, est destinée au dépôt et à la diffusion de documents scientifiques de niveau recherche, publiés ou non, émanant des établissements d'enseignement et de recherche français ou étrangers, des laboratoires publics ou privés. 


\title{
A Belief Function Theory Based Approach to Combining Different Representation of Uncertainty in Prognostics
}

\author{
Piero Baraldi ${ }^{\mathrm{a}}$, Francesca Mangili ${ }^{\mathrm{b}}$, Enrico Zio ${ }^{\mathrm{c}, \mathrm{a}}$ \\ ${ }^{a}$ Dipartimento di Energia, Politecnico di Milano, Italy \\ b"Dalle Molle" Institute for Artificial Intelligence (IDSIA), USI -SUPSI, Lugano (Switzerland) \\ 'Chair on Systems Science and the Energetic challenge, European Foundation for New Energy-Electricite' \\ de France, Ecole Centrale Paris and Supelec, France
}

\begin{abstract}
In this work, we consider two prognostic approaches for the prediction of the remaining useful life (RUL) of degrading equipment. The first approach is based on Gaussian Process Regression (GPR) and provides the probability distribution of the equipment RUL; the second approach adopts a Similarity-Based Regression (SBR) method for the RUL prediction and belief function theory for modeling the uncertainty on the prediction. The performance of the two approaches is comparable and we propose a method for combining their outcomes in an ensemble. The least commitment principle is adopted to transform the RUL probability density function supplied by the GPR method into a belief density function. Then, the Dempster's rule is used to aggregate the belief assignments provided by the GPR and the SBR approaches. The ensemble method is applied to the problem of predicting the RUL of filters used to clean the sea water entering the condenser of the boiling water reactor (BWR) in a Swedish nuclear power plant. The results by the ensemble method are shown to be more satisfactory than that provided by the individual GPR and SBR approaches from the point of view of the representation of the uncertainty in the RUL prediction.
\end{abstract}

Keywords: Prognostics, uncertainty representation, belief function theory, Gaussian process regression, filter clogging

\section{Introduction}

For industry, unforeseen equipment failures are extremely costly in terms of repair costs and lost revenues. To anticipate failures, predictive maintenance approaches are being developed, based on the assessment of the actual equipment degradation condition and on the prediction of its evolution for setting the optimal time for maintenance $[22,23,44,48,49]$. The underlying concept is that of failure prognostics, i.e., predicting the Remaining Useful Life (RUL) of the equipment, defined as the amount of time it will continue to perform its function according to the design specifications. 
In this work we tackle the problem of predicting the RUL of filters placed upstream the condenser of the boiling water reactor (BWR) of a Swedish nuclear power plant. The filters main function is to clean the sea water entering the secondary side of the cooling system. During operations, particles, seaweed, and mussels from the cooling water can cumulate in the filter medium, causing a clogging process. Thus, to assure correct and efficient operations, which require stopping these wastes before entering the condenser, prompt and effective cleaning of the filter is desirable. In this respect, predictive maintenance can allow to increase the component reliability, keeping maintenance costs reasonably low.

We consider a case in which few sequences of observations taken during the clogging process experienced by filters of the same plant in the past are available (training trajectories). Each observation contains the values of three parameters (pressure drop, flow across the filter and sea water temperature), which provide indirect indications about the degradation (clogging) state of the filters. Since the clogging process under investigation is affected by large uncertainties, mainly due to the variable conditions of the sea water, the challenge is to associate a confidence interval to the RUL prediction. This uncertainty assessment, which describes the expected mismatch between the real and predicted equipment failure times, can be used by the maintenance planner to confidently plan maintenance actions, according to the desired risk tolerance $[3,19$, $36,38,39]$. Thus, a proper characterization and representation of the uncertainties affecting the RUL prediction is of paramount importance in prognostics.

Given the unavailability of an explicit model of the degradation process, we resort to data-driven methods for RUL predictions. Among data-driven methods one can distinguish between (i) degradation-based approaches, modeling the future equipment degradation evolution and (ii) direct RUL prediction approaches, directly predicting the RUL [45].

Degradation-based approaches (i) are based on statistical models that learn the equipment degradation evolution from time series of the observed degradation states [16, 44, 47]; the predicted degradation state is, then, compared with failure criteria, such as the value of degradation beyond which the equipment fails performing its function (failure threshold). Examples of modeling techniques used in degradation-based approaches are Auto-Regressive models [10, 16], multivariate adaptive regression splines [18], Artificial Neural Networks [23, 24], Relevance Vector Machines [26] and Gaussian Processes [4,31].

Direct RUL predictions approaches (ii), instead, typically resort to artificial intelligence techniques that directly map the relation between the observable parameters and the equipment RUL, without the need of predicting the equipment degradation state evolution and fixing a failure threshold [27,34,50]. Examples of techniques used in direct RUL prediction approaches are the Bayesian approach [25] and similarity measures $[3,50]$.

Degradation-based prognostics provides more informative and transparent outcomes than direct RUL prediction prognostics, since it supplies a prediction not only of the current equipment RUL, but also of the entire degradation trajectory that the equipment will follow. This can be very useful, since it allows checking the prediction consistency considering expert intuition or information on-line acquired during the equipment degradation. However, degradation-based prognostics, differently from direct RUL prediction prognostics, requires identifying a degradation indicator and fixing a failure threshold, which could not be easy in practice and may introduce further uncertainty and sources of errors. 
Since in practice, it is often hard to choose between degradation-based and direct RUL prediction prognostics, in this work we consider the possibility of aggregating the predictions of a degradation-based and a direct RUL prediction method. This choice is also motivated from the observation that the aggregation of the outcomes of multiple models built using different pieces of information and different modeling approaches has been proven to make the prediction more accurate and robust [29].

In this context, the main contribution of the present work is to propose a technique for aggregating the outcomes provided by different prognostic approaches taking into account the prediction uncertainty.

With respect to the degradation-based approaches (type (i)), we have adopted Gaussian Process Regression (GPR) $[4,31]$ to fit the degradation probability distribution function (pdf) to the degradation trajectories of training. The uncertainty in the future evolution of the degradation states is explicitly modeled by GPR and the predictions about the future degradation state distribution is provided in the form of a Gaussian pdf [4, 24]. Finally, by comparing the predicted distribution of the future degradation states with a failure threshold, we estimate the probability distribution of the equipment RUL and the desired prediction intervals. The choice of GPR is motivated by the fact that other regression methods such as ANNs, which in recent research works have been shown to provide accurate predictions of the degradation state $[43,15]$, typically do not provide an explicit and direct quantification of the uncertainty of the predicted degradation states, as do methods like Relevance Vector Machine (RVM) [40] or Gaussian Process Regression (GPR) [20, 31]. Since the RVM method is actually a special case of a Gaussian Process (GP) [31], GPR has been used in this work. With respect to the direct RUL prediction approaches (type (ii)), we have adopted an approach based on the combined use of Similarity-Based Regression (SBR) [50] and Belief Function Theory (BFT) (also called Dempster-Shafer or evidence theory [14, 35]). Similarity-based regression methods are able to provide reliable RUL predictions even in a case, such as the one here addressed, in which very few training degradation trajectories are available [3]. Notice that other direct RUL prediction approaches, such as the Bayesian approach proposed in [25], typically require the availability of a larger number of training trajectories in order to provide reliable RUL predictions. Our estimate of the prediction uncertainty is, then, based on the use of Belief Function Theory (BFT). This choice is motivated by the large amount of uncertainty to which the model predictions are expected to be subject, given the randomness of the degradation process and the large imprecision of the employed empirical models trained using only the few available degradation trajectories. Indeed, according to the considerations in [5, 17, 41], it has been argued that the representation of the RUL uncertainty using probability distributions could be critical and uncertainty representation is best accounted for by Belief Function Theory. The result of the application of the method is a Basic Belief Assignment (BBA) that quantifies one's belief about the value of the test trajectory RUL, given the evidence provided by the reference trajectories. The identification of prediction intervals relies on the definition of the total belief assigned by the predicted BBA to an interval, which is interpreted as a lower bound for the probability that the test equipment RUL belongs to such interval.With respect to the aggregation of the predictions of the two approaches, the problem is complicated by the necessity of taking into account the prediction uncertainty representations provided by the two methods. Thus, techniques for the aggregation of point predictions, ranging from statistical methods, such as the mean and the median [7,30], to weighed average based on global or local performance measures of the individual models [1, 11], are not considered in this work since they do not take into account uncertainty. Similarly, 
techniques for the combination of individual probability distributions into a single aggregated probability distribution [12] cannot be used given that the two prognostic approaches provide different representations of the RUL uncertainty, i.e. the GPR method provides the RUL pdf, whereas the SBR provides the RUL BPA. A possibility to aggregate these two different uncertainty representations could be to transform the BPA into a probability distribution by applying a proper transformation technique such as the pignistic transformation proposed by Smeth [37]. Since a probability distribution is much more informative than a BBA, a limitation of this approach is that it would inject information that is actually not present available into the uncertainty representation. Thus, in order to perform the aggregation, we resort to the extension of the BFT to the continuous real axis $\Re$ [35], which allows transforming the belief functions into belief densities. Then, using the least commitment principle, the RUL pdf predicted by the GPR method is transformed into a belief density function and, finally, the Dempster's rule of combination is applied to aggregate the BBAs provided by the similarity-based approach and the GPR method $[2,32,46]$.

The remaining part of the paper is organized as follows: in Section 2, we briefly state the prognostic problem of interest; Section 3 describes the method for performing RUL predictions based on GPR; in Section 4, the methodology for providing prediction intervals for the RUL value based on the SBR method in the framework of BFT is described; in Section 5 a BFT-based technique for aggregating the outcomes of the GPR and SBR approaches is proposed; Section 6 presents the results of the numerical application of these methods to the prediction of the RUL for clogging filters; finally, in Section 7 we state our conclusions and suggest some potential future works.

\section{Problem statement}

We assume that a set of trajectories containing measurements collected during the process of degradation of $R$ pieces of equipment similar to the one of interest (test equipment) is available for training. Each training trajectory $r=1: R$ is made of a sequence $\mathrm{z}_{1: n^{r}}^{r}$ of observations $\mathbf{z}_{j}^{r}=\left[z_{1}^{r}\left(\tau_{j}^{r}\right), \ldots, z_{p}^{r}\left(\tau_{j}^{r}\right), \ldots, z_{P}^{r}\left(\tau_{j}^{r}\right)\right]$ representing the evolution of $P$ relevant parameters $z_{p}$ measured at time instants $\tau_{j}^{r}, j=1: n^{r}$ during the degradation evolution of the $r$-th equipment, up to the last measurement time $\tau_{n^{r}}^{r}$ before its failure occurring at time $\tau_{F}^{r}$. It is also assumed that from the values of the parameters in the observation $\mathrm{z}_{j}^{r}$ we can derive an indication $z_{\delta, j}^{r}$ about the degradation state $\Delta_{j}^{r}$ of the $r$-th equipment at time $\tau_{j}^{r}$. An equipment is assumed to fail when its indication of degradation exceeds the maximum acceptable degradation state $\delta^{\text {th }}$, called failure threshold. A sequence of observations $\mathbf{z}_{1: J}^{\text {test }}$ from $\tau_{1}^{\text {test }}$ to the present time $\tau_{J}^{\text {test }}$, and consequently a sequence of degradation indications, $z_{\delta, 1: J}^{\text {test }}$ derived from the observations $\mathbf{z}_{1: J}^{\text {test }}$, are available also for the test equipment.

The goal of the prognostics model is to predict the RUL of the test equipment at the present time $\tau_{J}$. Due to the variability of several factors influencing the degradation process such as the microstructural and manufacturing equipment characteristics and the loading and external conditions, the degradation evolution is typically represented by a stochastic process [24]. As a consequence, the degradation state of the equipment at any time $\tau$ and its RUL at the present time $\tau_{J}^{\text {test }}$ are random variables $\Delta(\tau)$ and $R U L_{J}^{\text {test }}$. Then, prognostics must provide not only a prediction $r u l_{J}$ of the expected value of the variable $R U L_{J}^{\text {test }}$ but also a measure of its uncertainty [9]. In this work, we represent the uncertainty on the RUL prediction in the 
form of a left bounded prediction interval $P I(\alpha)=\left[r u l_{J}^{\text {inf }},+\infty\right)$ containing the true value of the test equipment RUL, hereafter referred to as $r u l_{J}^{\text {true }},{ }^{r u l_{J}^{\text {true }}}$, with probability at least equal to $\alpha$. The left bound of the RUL, $r u l_{j}^{\text {inf }}(\alpha)$, can be used by the maintenance planner to decide the time at which the next maintenance intervention should be performed, ensuring that the maximum acceptable probability of failure is not exceeded.

\section{Degradation-based prognostics: Gaussian Process Regression}

Within a degradation-based prognostic approach, we aim to model the evolution of the equipment degradation as a stochastic process based on the available observations $z_{\delta, 1: n^{r}}^{r}$ of the degradation evolution of similar pieces of equipment. The obtained distribution of the future degradation states is then compared with the failure threshold $\delta^{\text {th }}$, whose value is assumed to be known, to predict the distribution of the equipment RUL.

GPR is a powerful and flexible approach for performing probabilistic inference over functions [31] and can be effectively used for modeling degradation as a stochastic process [4]. To do that, it is necessary to assume that the distribution of the degradation states is Gaussian with different mean $\bar{\Delta}(\tau)$ and variance $\sigma_{\Delta}^{2}(\tau)$ at each time instant $\tau$; then, the GPR method is used to evaluate the conditional probability density function (pdf) $p_{\Delta\left(\tau^{\text {test }}\right)}\left(\delta^{\text {test }} \mid \mathbf{D}_{\tau / z}^{+}\right)$of the future degradation states $\Delta\left(\tau^{\text {test }}\right), \tau^{\text {test }}>\tau_{J}^{\text {test }}$ of the test trajectory, given the observation dataset $\mathbf{D}_{\tau / z}^{+}$containing the dataset $\mathbf{D}_{\tau / z}^{\text {train }}=\left\{\left(\tau_{j}^{r} ; z_{\delta, j}^{r}\right)_{j=1: n^{r} ; r=1: R}\right\}$ drawn from the training trajectories and the dataset $\mathbf{D}_{\tau / z}^{\text {test }}=\left\{\left(\tau_{j}^{\text {test }} ; z_{\delta, j}^{\text {test }}\right)_{j=1: J}\right\}$ drawn from the test trajectory. For mapping the function $\Delta(\tau)$ given the input $\tau$, GPR defines the prior in the form of a distribution over functions specified by a Gaussian Process (GP) [20, 31]. A GP is a collection of random variables any finite number of which has a joint Gaussian distribution. A real GP $\Delta(\tau)$ is completely specified by its mean function $m_{\Delta}(\tau)$ and covariance function $k_{\Delta}\left(\tau, \tau^{\prime}\right)$ :

$$
\begin{aligned}
& \Delta(\boldsymbol{\tau}) \sim \mathrm{GP}\left\{m_{\Delta}(\boldsymbol{\tau}) ; K_{\Delta}(\boldsymbol{\tau}, \boldsymbol{\tau})\right\} \\
& m(\tau)=E[\Delta(\tau)] \\
& k\left(\tau, \tau^{\prime}\right)=E\left[\left(\Delta(\tau)-m_{\Delta}(\tau)\right)\left(\Delta\left(\tau^{\prime}\right)-m_{\Delta}\left(\tau^{\prime}\right)\right)\right]
\end{aligned}
$$

where $\tau$ represents a vector of input values and $K_{\Delta}(\tau, \tau)$ indicates the co-variance matrix containing the values of $k\left(\tau, \tau^{\prime}\right)$ evaluated for all possible pairs of inputs in $\tau$.

This prior is taken to represent our prior beliefs over the kind of functions we expect to observe. Typically the prior mean and co-variance functions depend from some free parameters usually called hyperparameters. Although the choice of the covariance function must be specified by the user, various methods have been proposed for determining the corresponding hyper-parameters from training data [31]. Here, the hyper-parameters are optimized by maximizing the marginal likelihood of the dataset set $\mathbf{D}_{\tau / z}^{\text {train }}$ drawn from the training trajectories using the conjugate gradient method.

Given the prior information about the GP, the set of hyper-parameters and the observation dataset $\mathbf{D}_{\tau / z}^{+}$, the posterior distribution is derived by imposing a restriction on the prior distribution to contain only those 
functions that agree with the observed data [31]. In other words, we condition the output in correspondence of the test input vector $\tau^{\text {test }}$ to the available observations $\mathbf{D}_{\tau / z}^{+}$drawn from the same GP, and thus we have:

$$
\left[\begin{array}{c}
\Delta\left(\boldsymbol{\tau}^{\text {train }}\right) \\
\Delta\left(\tau^{\text {test }}\right)
\end{array}\right] \sim \operatorname{GP}\left\{\left[\begin{array}{c}
m_{\Delta}\left(\boldsymbol{\tau}^{\text {train }}\right) \\
m_{\Delta}\left(\tau^{\text {test }}\right)
\end{array}\right] ;\left[\begin{array}{cc}
K_{\Delta}\left(\boldsymbol{\tau}^{\text {train }}, \boldsymbol{\tau}^{\text {train }}\right) & K_{\Delta}\left(\boldsymbol{\tau}^{\text {train }}, \tau^{\text {test }}\right) \\
K_{\Delta}\left(\tau^{\text {test }}, \boldsymbol{\tau}^{\text {train }}\right) & K_{\Delta}\left(\tau^{\text {test }}, \tau^{\text {test }}\right)
\end{array}\right]\right\}
$$

where $\boldsymbol{\tau}^{\text {train }}$ is the vector of all the inputs in $\mathbf{D}_{\tau / z}^{+}$..

The posterior distribution of the output $\Delta\left(\tau^{\text {test }} \mid \mathbf{D}_{\tau / z}^{+}\right)$in correspondence of the input $\tau^{\text {test }}$ is Gaussian with mean $\bar{\Delta}\left(\tau^{\text {test }}\right)$ and variance $\sigma_{\Delta}^{2}\left(\tau^{\text {test }}\right)$ which can be derived from eq. (2) [31]:

$$
\Delta\left(\tau^{\text {test }} \mid \mathbf{D}_{\tau / z}^{+}\right) \sim N\left(\bar{\Delta}\left(\tau^{\text {test }}\right), \sigma_{\Delta}^{2}\left(\tau^{\text {test }}\right)\right)
$$

where

$$
\begin{aligned}
& \bar{\Delta}\left(\tau^{\text {test }}\right)=m_{\Delta}\left(\tau^{\text {test }}\right)+K\left(\boldsymbol{\tau}^{\text {train }}, \tau^{\text {test }}\right)\left[K\left(\boldsymbol{\tau}^{\text {train }}, \boldsymbol{\tau}^{\text {train }}\right)\right]^{-1}\left(\mathbf{z}^{\text {train }}-m_{\Delta}\left(\boldsymbol{\tau}^{\text {train }}\right)\right) \\
& \sigma_{\Delta}^{2}\left(\tau^{\text {test }}\right)=k_{\Delta}\left(\tau^{\text {test }}, \tau^{\text {test }}\right)-K_{\Delta}\left(\tau^{\text {test }}, \boldsymbol{\tau}^{\text {train }}\right)\left[K_{\Delta}\left(\boldsymbol{\tau}^{\text {train }}, \boldsymbol{\tau}^{\text {train }}\right)\right]^{-1} K_{\Delta}\left(\boldsymbol{\tau}^{\text {train }}, \tau^{\text {test }}\right)
\end{aligned}
$$

where $\mathbf{z}^{\text {train }}$ is the vector of all the outputs in $\mathbf{D}_{\tau / z}^{+}$.

Since the data $\mathbf{D}_{\tau / z}^{+}$, which are used for conditioning the prior GP, are originated from both the training and test equipments, the GPR can learn the structure underlying the degradation processes and the specific variation around this structure that specifically characterizes the test trajectory. This result is obtained by using a covariance function of the following form [4, 21]:

$$
k\left(\tau_{j}^{r}, \tau_{j^{\prime}}^{r^{\prime}}\right)=k_{1}\left(\tau_{j}^{r}, \tau_{j^{\prime}}^{r^{\prime}}\right)+k_{2}\left(\tau_{j}^{r}, \tau_{j^{\prime}}^{r^{\prime}}\right) \delta\left(r, r^{\prime}\right)+\sigma_{z}^{2} \delta\left(r, r^{\prime}\right) \delta\left(j, j^{\prime}\right)
$$

where $\sigma_{z}^{2}$ is the variance of the white Gaussian noise affecting the observations $z_{\delta, j}^{r}$ and the reference index assigned to the test trajectory is $r=R+1$. The first term of the kernel corresponds to the covariance associated with the common structure underlying all degradation trajectories; the second represents the covariance owing to the variation of each trajectory around the common structure of all degradation trajectories. This term assumes a finite value only when $\tau_{j}^{r}$ and $\tau_{j}^{r^{\prime}}$ are taken from the same trajectory, since we assume the variation specific to each trajectory to be uncorrelated across trajectories. Finally, the third term accounts for the observation noise associated with the observation $z_{\delta, j}^{r}$ of the degradation state $\delta_{j}^{r}$.

Given the known value of the failure threshold and the conditional distribution of the degradation state $p_{\Delta\left(\tau_{\text {test }}\right)}\left(\delta \mid \mathbf{D}_{\tau / z}^{+}\right)$, the RUL cumulative distribution function (cdf) $P_{R U L_{J}^{\text {est }}}\left(r u l_{J} \mid \mathbf{D}_{\tau / z}^{+}\right)$is computed as the probability that the degradation $\Delta\left(\tau^{\text {test }}\right)$ at the future time $\tau^{\text {test }}=\tau_{J}^{\text {test }}+r u l_{J}$ will exceed the failure threshold $d_{t h}[3]$ : 


$$
\begin{gathered}
P_{R U L\left(\tau_{J}\right)}\left(r u l_{J} \mid \mathbf{D}_{\tau / z}^{+}\right)=\operatorname{Prob}\left(R U L_{J}^{\text {test }}<r u l_{J} \mid \mathbf{D}_{\tau / z}^{+}\right) \\
=\int_{d^{t h}}^{+\infty} p_{\Delta\left(\tau_{J}^{\text {test }}+r u l_{J}\right)}\left(\delta(\tau) \mid \mathbf{D}_{\tau / z}^{+}\right) \mathrm{d} \delta==1-\Phi\left(\frac{d^{\text {th }}-\bar{\Delta}\left(t_{J}^{\text {test }}+r u l_{J}\right)}{\sigma_{\Delta}\left(\tau_{J}^{\text {test }}+r u l_{J}\right)}\right)
\end{gathered}
$$

where $\Phi$ is the standard normal cdf.

From the RUL cdf one can derive the prediction $r \hat{u} l_{J}^{G P R}$ of the equipment RUL as the expected value of the RUL distribution and the left bounded prediction interval $C I_{J}^{G P R}(\alpha)=\left[r u l_{J}^{\text {inf,GPR }}(\alpha),+\infty\right]$ as the interval containing with probability $\alpha$ the true value of the test equipment RUL, hereafter referred to as $r u l_{J}^{\text {true }}$.

\section{Direct RUL prediction: Similarity-based RUL prediction}

Within a direct RUL prediction prognostic approach, the mapping between observations $\mathbf{z}_{j}^{r}$ (or sequences of observations) and the corresponding RUL value is derived directly from the training trajectories without modeling the degradation process. In this work, this is done by using the similarity-based regression model presented in [3]. The idea underpinning this approach is to evaluate the similarity between the test trajectory and $R$ available reference trajectories, and to use the RULs of these latter to estimate the RUL of the former, considering how similar they are $[28,45,50]$.

The approach requires to define a measure of similarity between trajectories. This is done considering the pointwise difference between $n$-long sequences of observations. At the present time, $\tau_{J}$, the distance $d_{j}^{r}$ between the sequence of the $n$ latest observations $\mathbf{z}_{J-n+1: J}^{\text {test }}$ of the test trajectory, and all $n$-long segment $\mathbf{z}_{j-n+1: j}^{r}, j=1: n^{r}$ of all reference trajectories $r=1: R$ is computed as:

$$
d_{j}^{r}=\sqrt{\sum_{i=1}^{n}\left\|\mathbf{z}_{J-n+i}^{t e s t}-\mathbf{z}_{j-n+i}^{r}\right\|^{2}}
$$

where $\|\mathbf{x}-\mathbf{y}\|^{2}$ is the square Euclidean distance between vectors $\mathbf{x}$ and $\mathbf{y}$.

The similarity $s_{j}^{r}$ of the training trajectory segment $\mathbf{z}_{j-n+1 ; j}^{r}$ to the test trajectory is defined as a function of the distance measure $d_{j}^{r}$. In [50], the following bell-shaped function has turned out to give robust results in similarity-based regression due to its gradual smoothness:

$$
s_{j}^{r}=\exp \left(-\frac{d_{j}^{r^{2}}}{\lambda}\right)
$$


The arbitrary parameter $\lambda$ can be set by the analyst to shape the desired interpretation of similarity: the smaller is the value of $\lambda$, the stronger the definition of similarity. A strong definition of similarity implies that the two segments under comparison have to be very close in order to receive a similarity value $s_{j}^{r}$ significantly larger than zero.

For the prediction of the test equipment RUL, a RUL value $r \hat{u} l_{j^{*}}^{r}$ is assigned to each training trajectory $r=1: R$ by considering the difference between the trajectory failure time $\tau_{F}^{r}$ and the last time instant $\tau_{j^{*}}^{r}$ of the trajectory segment $\mathbf{z}_{j^{*}-n+1: j^{*}}^{r}$ which has the maximum similarity $s_{j^{*}}^{r}$ with the test trajectory:

$$
r \hat{u} l_{j^{*}}^{r}=\tau_{F}^{r}-\tau_{j^{*}}^{r}
$$

Then, the prediction $r \hat{u} l_{J}^{S B}$ of the test equipment RUL at time $\tau_{J}^{\text {test }}$ is given by the similarity-weighed sum of the values $r u l_{j^{*}}^{r}$ :

$$
r \hat{u} l_{J}^{S B}=\frac{\sum_{r=1}^{R} s_{j^{*}}^{r} r \hat{u} l_{j^{*}}^{r}}{\sum_{r=1}^{R} s_{j^{*}}^{r}}
$$

Given the intrinsic randomness of the degradation process and the prediction errors performed by the empirical model, it is important to associate the point predictions provided by eq. (10) with a quantification of its uncertainty. In particular, since in this work we consider situations characterized by degradation processes affected by large uncertainties and we use empirical models developed using few degradation trajectories, we expect RUL predictions characterized by very large uncertainty. In this context, a nonprobabilistic uncertainty representation method, the Belief Function Theory (BFT) [14,35], has been adopted for uncertainty representation given its capability of representing very limited knowledge $[5,17,41]$. If we consider, for example, an extreme case, in which the only information available on the equipment RUL is that it will fail in the time interval $\left[0, \tau_{F}^{\max }\right]$, the classical probabilistic representation of the uncertainty is provided by a uniform distribution with range $\left[0, \tau_{F}^{\max }\right]$, according to the principle of indifference. However, as it has been shown in [46], this assignment causes the paradox that it assigns a precise probability value to an event such as "RUL in the interval $\left[0, \tau_{F}^{\max } / 2\right]$ ", whereas, according to the available knowledge, the probability of this event can have any value between 0 and 1 . For these reasons, in cases characterized by the large uncertainty, we prefer the use of an approach based on the Belief Function (or Dempster-Shafer) theory (BFT). Few notions of BFT will be recalled in the paper, when necessary for the comprehension of the method. For a general introduction to the BFT and for further details about the mathematical developments and interpretations of the theory, the interested reader is referred to [14, 35, 36].

The belief about the value of an uncertain variable $X$ is represented by a basic belief assignment (BBA), which assigns to subsets, $Y^{i}$, of the domain of $X$ (called frame of discernment, $\Omega_{X}$, in the BFT terminology) a mass $m_{X}\left(Y^{i}\right)$ based on the available information. All the subsets $Y^{i}$ of $\Omega_{X}$ with associated a mass $m_{X}\left(Y^{i}\right)>0$ are referred to as focal sets. The BBA should verify the condition that the sum of the masses of all its focal elements is 1 . In our prognostic problem, the uncertain variable is the equipment RUL 
at the present time, $R U L_{J}^{\text {test }}$, whose frame of discernment $\Omega_{R U L_{J}^{\text {test }}}$ is defined as the interval $\left[0, \tau_{F}^{\max }-\tau_{J}^{\text {test }}\right]$, where $\tau_{F}^{\max }$ is the maximum possible life duration of the equipment.

We assume that each reference trajectory, $\mathbf{z}_{1: n^{r}}^{r}, r=1, \ldots, R$, corresponds to a different "expert" ("agent", BTF terminology) and that each expert provides a BBA assignment defined by only one focal set $\left\{r \hat{u} l_{j^{*}}^{r}\right\}$ with associated mass $m_{R U L_{J}^{\text {lest }}}^{r}\left(\left\{r \hat{u} l_{j^{*}}^{r}\right\}\right)=1, r=1: R$.

Following the approach used in [28], the similarity measure $s_{j}^{r}$ defined in eq. (8) is interpreted as a measure of the relevance of the source of information inducing the BBA $m_{R U L_{J}^{t e s t}}^{r}\left(\left\{r \hat{u} l_{j^{*}}^{r}\right\}\right)=1$ and the discounting operation is used to reduce the belief assigned to the $r$-th expert $m_{R U L_{J}^{t e s t}}^{r}$ to $\left\{r \hat{u} l_{j^{*}}^{r^{*}}\right\}$ by a factor $\left(1-\gamma \cdot s_{j^{*}}^{r}\right)$, with $\gamma \in[0,1]$, representing the dissimilarity between the test and the $r$-th training trajectory. The discounted BBAs $\tilde{m}_{R U L_{J}^{t s t}}^{r}\left(\left\{r \hat{u} l_{j^{*}}^{r}\right\}\right), r=1: R$, are thus obtained [28]:

$$
\begin{aligned}
& \tilde{m}_{R U L_{J}^{\text {test }}}^{r}\left(\left\{r \hat{u} l_{j^{*}}^{r}\right\}\right)=\gamma \cdot s_{j^{*}}^{r} \\
& \tilde{m}_{R U L_{J}^{\text {est }}}^{r}\left(\Omega_{R U L_{J}^{\text {test }}}\right)=1-\gamma \cdot s_{j^{*}}^{r}
\end{aligned}
$$

The arbitrary parameter $\gamma \in[0,1]$ defines the degree of trust given to the reference trajectories. The mass assigned to the frame of discernment $\Omega_{R U L_{J}^{t e s t}}$ represents the ignorance about the value of $R U L_{J}^{\text {test }}$, because it indicates the absence of evidence that the value of $R U L_{J}^{\text {test }}$ belongs to any subset of $\Omega_{R U L_{J}^{\text {est }}}$. It is important to notice that $\gamma<1$ implies that a part of belief is assigned to the ignorance represented by $\Omega_{R U L}$, even in the unrealistic case of a reference trajectory exactly identical to the test one. For a detailed discussion about the choice of the values of the parameters $\gamma$ and $\lambda$, the interested reader is referred to [3].

The information provided by each trajectory, modeled by the BBA in eq. (11), needs to be aggregated to provide the RUL prediction. The pioneering approach to the aggregation of multiple pieces of evidence was suggested by Dempster [14] and has become the standard way of combining multiple BBAs. In the following, we first describe the Dempter's rule of combination for the case of two belief structures. According to this rule, two distinct sources of information inducing two BBAs, e.g., $\tilde{m}_{R U L_{J}^{\text {lest }}}^{1}$ and $\tilde{m}_{R U L_{J}^{\text {lest }}}^{2}$, with corresponding focal elements $Y_{1}^{j_{1}}$ and $Y_{2}^{j_{2}}$, can be combined to obtain the aggregated BBA $m_{R U L_{I}^{1 \oplus s t}}^{1 \oplus J}$ with focal sets $Y^{j}$ given by all non-empty intersections of pairs of focal elements $Y_{1}^{j_{1}}$ and $Y_{2}^{j_{2}}$. Each focal set $Y^{j}$ receives a mass of belief equal to [28]:

$$
m_{R U L_{J}^{\text {sest }}}^{1 \oplus 2}\left(Y^{j}\right)=\frac{1}{K} \sum_{Y_{1}^{j_{1}} \cap Y_{2}^{j_{2}}=Y^{j}} \tilde{m}_{R U L_{J}^{\text {sest }}}^{1}\left(Y_{1}^{j_{1}}\right) \tilde{m}_{R U L_{J}^{\text {sest }}}^{2}\left(Y_{2}^{j_{2}}\right)
$$

where $K$ is a normalization factor introduced to ensure that, while restricting the focal elements of $m_{R U L_{J}^{\text {ket }}}^{1 \oplus 2}\left(Y^{j}\right)$ to be non-empty sets, we still obtain that the sum of the masses equals one. Notice that the mass that would be assigned to the empty set by applying eq. (12) without the normalization term $K$ represents the conflicting information. The choice of removing this mass and re-normalizing the resulting BBA has raised some concern and criticism. This has inspired alternative methods of normalization; see, for example, Yamada [42] for a comprehensive discussion of these issues. The necessary exploration of alternative 
approaches for handling the belief associated to the conflicting information in the aggregation of multiple BBAs is left to future work.

In the case of multiple belief structures, $\tilde{m}_{R U L_{J}^{t e s t}}^{i}$ for $i=1, \ldots, R$, each one having focal set $Y_{i}^{j}$, the Dempter's rule of combination can be easily extended. In particular, each focal set $Y$ of the aggregated BBA $m_{R U L_{J}^{t e s t}}$ is obtained as the non-null intersection of one focal set $Y_{i}{ }^{j_{i}}$ from each of the contributing BBAs, and its associated mass of belief is

$$
m_{R U L_{J}^{\text {est }}}\left(Y^{j}\right)=\frac{1}{K} \prod_{i=1}^{R} \tilde{m}_{R U L_{J}^{i s t}}^{i}\left(Y_{i}^{j_{i}}\right)
$$

Then, by applying eq. (13) to the $R$ discounted BBAs $\tilde{m}_{R U L_{J}^{\text {lest }}}^{r}$ in eq. (11), one obtains the aggregated BBA [28] which has as focal sets the degenerate intervals $\left\{r \hat{u} l_{j^{*}}^{r}\right\}$ and the frame of discernment $\Omega_{R U L_{J}^{\text {test }}}$. The focal sets $\left\{r \hat{u} l_{j^{*}}^{r}\right\}, r=1: R$, are obtained as the intersection of the focal set $\left\{r \hat{u} l_{j^{*}}^{r}\right\}$ for the BBA corresponding to the $r$-th trajectory and the focal sets represented by the frame of discernment $\Omega_{R U L_{J}^{e s t}}$ for the other trajectories. Thus, the mass assigned to the focal set $\left\{r \hat{u} l_{j^{*}}^{r}\right\}$ of the aggregation structure is:

$$
m_{R U L_{J}^{t e s t}}\left(\left\{r \hat{u} l_{j^{*}}^{r}\right\}\right)=\frac{\gamma \cdot s_{j^{*}}^{r}}{K} \prod_{r^{\prime} \neq r}\left(1-\gamma \cdot s_{j^{*}}^{r^{\prime}}\right)
$$

The focal set $\Omega_{R U L_{J}^{t s t}}$ is obtained as the intersection for all the trajectories of the frame of discernment $\Omega_{R U L_{J}^{t e s t}}$ and, thus, its mass is:

$$
m_{R U L_{J}^{\text {test }}}\left(\Omega_{R U L_{J}^{\text {test }}}\right)=\frac{1}{K} \prod_{r^{\prime}=1}^{R}\left(1-\gamma \cdot s_{j^{*}}^{r^{\prime}}\right)
$$

The normalization constant $K$ is obtained from the condition:

$$
\frac{1}{K} \prod_{r^{\prime}=1}^{R}\left(1-\gamma \cdot s_{j^{*}}^{r^{\prime}}\right)+\sum_{r=1}^{R} \frac{\gamma \cdot s_{j^{*}}^{r}}{K} \prod_{r^{\prime} \neq r}\left(1-\gamma \cdot s_{j^{*}}^{r^{\prime}}\right)=1 .
$$

The information conveyed by a BBA can be represented by a belief $\operatorname{Bel}_{f}\left(Y^{i}\right)$ or by a plausibility function $\mathrm{Pl}_{f}\left(Y^{i}\right)$ defined, respectively, as [41]

$$
\operatorname{Bel}_{f}\left(Y^{i}\right)=\sum_{Y^{i^{i} \subseteq Y^{i}}} m_{R U L_{J}}\left(Y^{i^{\prime}}\right)
$$

and

$$
\mathrm{Pl}_{f}\left(Y^{i}\right)=\sum_{Y^{i^{\prime}} \cap Y^{i} \neq \varnothing} m_{R U L_{J}}\left(Y^{i^{\prime}}\right)
$$


The belief associated to an interval $\left[R U L_{J}^{\mathrm{inf}}, R U L_{J}^{\text {sup }}\right]$ represents the amount of belief that directly supports the hypothesis $R U L_{J}^{\text {test }} \in\left[r u l_{J}^{\text {inf }}, r u l_{J}^{\text {sup }}\right]$, whereas the plausibility represents the maximum belief that could be committed to this hypothesis if further information became available [6,35,34]. Then, belief and plausibility can be seen as lower and upper bounds of the probability that the hypothesis $R U L_{J}^{\text {test }} \in\left[r u l_{J}^{\mathrm{inf}}, r u l_{J}^{\text {sup }}\right]$ is true. Let us consider a left bounded interval $\left[r u l_{J}^{\mathrm{inf}},+\infty\right]$ : the belief assigned to such interval is the lower bound of the probability that the RUL of the test equipment is larger than the left bound $r u l_{J}^{\mathrm{inf}}$. Thus, if the maintenance planner defines the maximum acceptable failure probability, $\alpha$, the method can provide a value $r u l_{j}^{\text {inf }}(\alpha)$ which guarantees that the test equipment will fail after $r u l_{j}^{\text {inf }}(\alpha)$ with a probability of, at least $\alpha$. The interval $C I_{J}^{S B}(\alpha)=\left[r u l_{J}^{\mathrm{inf}, S B}(\alpha),+\infty\right]$ will be referred to as left bounded prediction interval with belief $\alpha$.

\section{RUL uncertainty aggregation}

In order to properly aggregate the prediction of the approaches described in Sections 3 and 4, two issues have to be addressed: the aggregation of i) the point predictions and ii) the corresponding uncertainty representations.

With respect to i), we consider the average of the point predictors $r \hat{u} l_{J}^{G P R}$ and $r \hat{u} l_{J}^{S B}$ :

$$
r \hat{u} l_{J}^{C o m b}=\frac{r \hat{u} l_{J}^{G P R}+r \hat{u} l_{J}^{S B}}{2}
$$

Other, more advanced techniques for ensemble aggregation of point predictions have been proposed in the literature, ranging from statistics methods like the mean and the median [7, 30], to weighed averages of the model outcomes based on the global or local performances of the individual models $[1,11])$. Since our main objective in this work is the estimation of the prediction uncertainty, these techniques will be object of future research work.

With respect to ii), the most popular solutions for the combination of probability distributions are based on weighted averages (the interested reader is referred to [12] for an extensive review of aggregation strategies). However, these techniques aggregate model predictions that are all in the form of probability distributions, whereas, in our case, the similarity-based prognostic approach provides a prediction in the form of a BBA. Thus, the main difficulty faced in the aggregation of the RUL predictions provided by the similarity-based and the GPR approaches concerns the combination of two different representations of the prediction uncertainty. A possibility to aggregate these two different uncertainty representations is to transform the BBA into a probability distribution by applying a proper transformation technique such as the pignistic transformation proposed by Smeth [37]. Since a probability distribution is much more informative than a BBA, a limitation of this approach is that it injects arbitrary information into the uncertainty representation. On the other side, the application of the GPR model is based on the assumption that the degradation state has a Gaussian distribution which is not completely justified by our prior knowledge. Furthermore, the application of a GPR model requires setting the prior mean and covariance functions without precise information on them. Therefore, the pdf predicted by the GPR cannot be considered as an exact 
representation of the equipment RUL distribution, but it should be considered affected by errors and approximations. Thus, instead of transforming the BBA predicted by the similarity-based approach into a pdf, we choose to represent the GPR prediction in the BFT framework and use the conjunctive rule for the combination of multiple BBAs.

To represent a pdf, which is defined on a continuous space, in the BFT framework we resort to the generalization of the BFT on the continuous real axis $\mathfrak{R}$ proposed by [37] which allows transforming belief masses into densities. This theory assumes that masses are only allocated to closed intervals $[\underline{x}, \bar{x}]$, which can be represented by points in the half-plane $(\underline{x}, \bar{x}) \in \mathfrak{R}^{2}: \underline{x} \leq \bar{x}$ (Figure 1). A belief density function (BDF) $f(\underline{x}, \bar{x})$ is defined on this half-plane, which assigns to each point $(\underline{x}, \bar{x})$ the mass $m_{x}([\underline{x}, \bar{x}])$ representing the evidence that the uncertain quantity $x \in[\underline{x}, \bar{x}]$.

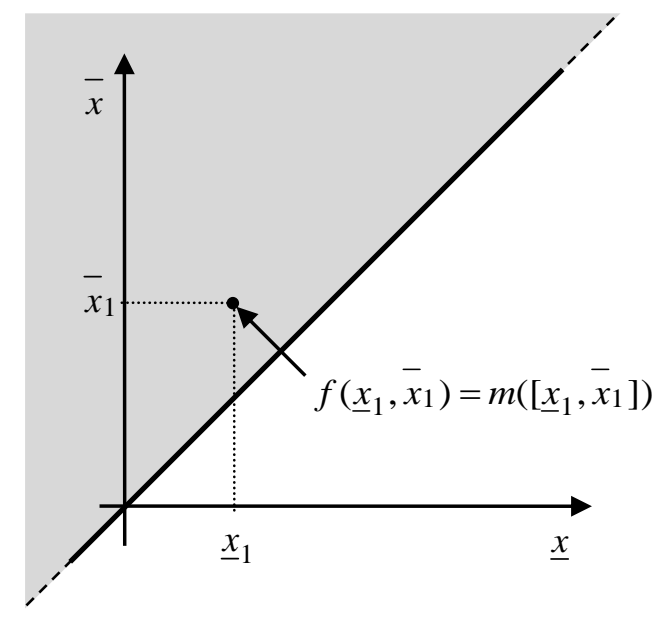

Figure 1: graphical representation of intervals $[\underline{x}, \bar{x}]$, modified from [37].

By extending eqs. (17) and (18) to a continuous domain, one can compute the belief and plausibility functions of any interval $\left[\underline{x}_{1}, \bar{x}_{1}\right]$. In particular, the total belief $\operatorname{Bel}_{f}\left(\left[\underline{x}_{1}, \bar{x}_{1}\right]\right)$ assigned by the BDF $f(\underline{x}, \bar{x})$ to an interval, e.g., $\left[\underline{x}_{1}, \bar{x}_{1}\right]$ represented by a point in Figure 1, is the integral of $f$ over the triangle $(\underline{x}, \bar{x}) \in \mathfrak{R}^{2}: \underline{x} \geq \underline{x}_{1}, \bar{x} \leq \bar{x}_{1}$ highlighted in grey in Figure 2 (left), whereas its plausibility $\mathrm{Pl}_{f}\left(\left[\underline{x}_{1}, \bar{x}_{1}\right]\right)$ is the integral of $f$ over the half-plane $(\underline{x}, \bar{x}) \in \mathfrak{R}^{2}: \underline{x} \leq \bar{x}_{1}, \bar{x} \geq \underline{x}_{1}$ highlighted in grey in Figure 2 (right). The interested reader may refer to $[32,37]$ for more details on the extension of the BFT to the continuous axis. 

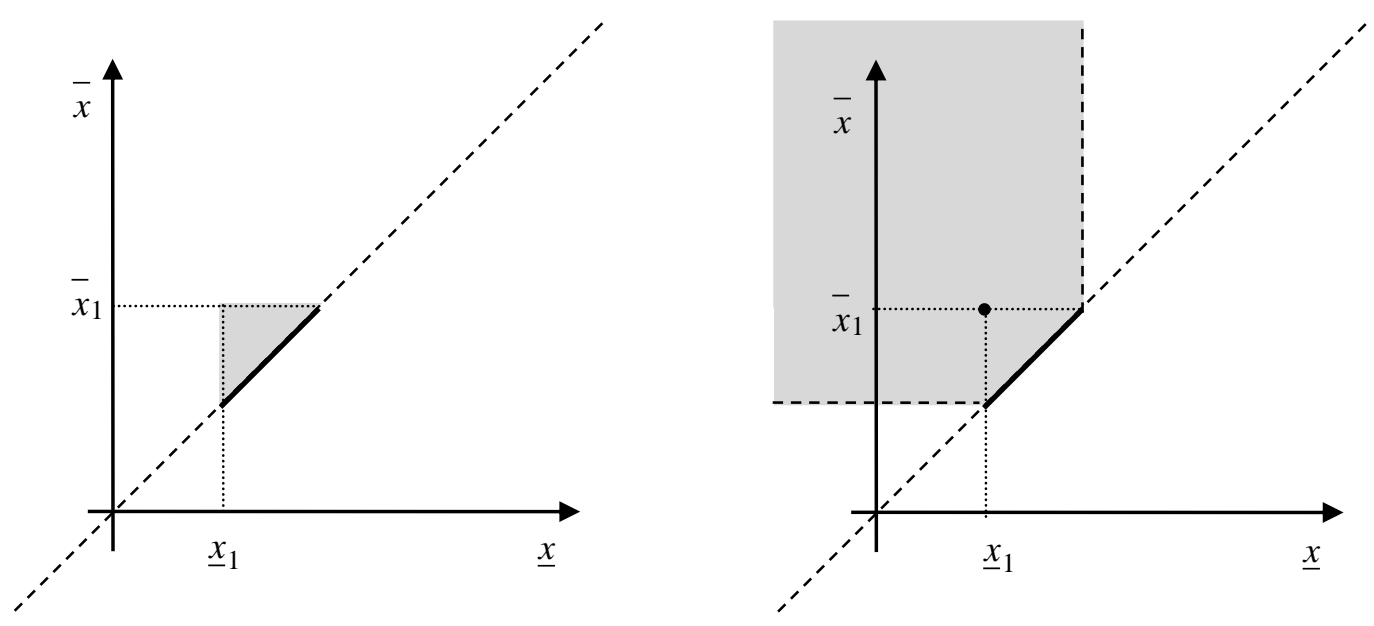

Figure 2: graphical representation of the belief (left) and plausibility (right) associated to the interval [ $\left.\underline{x}_{1}, \bar{x}_{1}\right]$, modified from [37].

The BBA generated by the similarity-based approach is characterized by the presence of a number $R$ of focal sets formed by a single point $\left\{r \hat{u} l_{j^{*}}^{r}\right\}$ and by some mass assigned to the entire frame of discernment $\Omega_{R U L_{J}^{\text {est }}}$ (eqs. (14) and (15)). Within the framework of continuous BFT, this BBA is transformed into a BDF by assigning finite masses only to the degenerated intervals of null dimension $\left[r \hat{u} l_{j^{*}}^{r}, r \hat{u} l_{j^{*}}^{r}\right], j=1: R$, lying on the boundary $\underline{R U L}=\overline{R U L}$ of the half-plane of all possible RUL intervals, and to the RUL domain $\Omega_{R U L_{J}^{t s t}}$, i.e., the interval $\left[0, R U L_{J}^{\max }\right]$, with $R U L_{J}^{\max }=\tau_{F}^{\max }-\tau_{J}$ (Figure 3). This BDF, indicated by $f_{R U L_{j}}^{S B}$, can be represented by:

$$
\begin{aligned}
f_{R U L_{j}}^{S B}(\underline{R U L}, \overline{R U L}) & =\sum_{r=1}^{R} m_{R U L_{J}^{\text {test }}}\left(\left\{r \hat{u} l_{j^{*}}^{r}\right\}\right) \cdot \delta_{\left(r \hat{u} l_{j^{*}}^{r}, r \hat{u} l_{j^{*}}^{r}\right)}(\underline{R U L}, \overline{R U L}) \\
& +m_{R U L_{J}^{\text {test }}}\left(\Omega_{R U L_{J}^{\text {est }}}\right) \cdot \delta_{\left(0, R U L_{J}^{\max }\right)}(\underline{R U L}, \overline{R U L})
\end{aligned}
$$

where $\delta_{(a, b)}$ is a Dirac delta functions which is always zero except when, respectively, the conditions $a=\underline{R U L}$ and $b=\overline{R U L}$ are verified. Notice that, since the equipment RUL assumes only positive values, the half-plane of interest for the RUL prediction (highlighted in grey in Figure 3) is defined by the two constraints $0 \leq \underline{R U L} \leq \overline{R U L}$. 


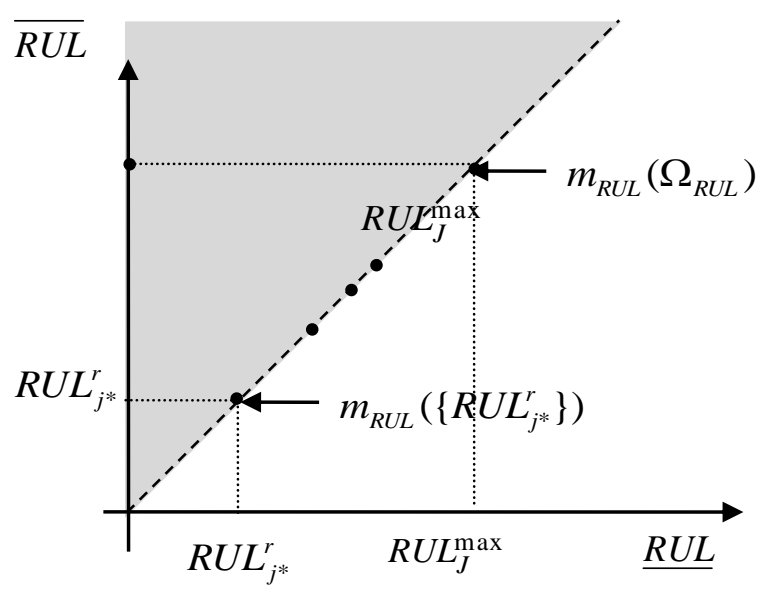

Figure 3: graphical representation of $f_{S B}(\underline{R U L}, \overline{R U L})$.

To represent the pdf predicted by the GPR in the BFT framework, we consider it as a representation of some potential betting behaviors, that is, a pignistic pdf Betf induced on $\mathfrak{R}$ by an underlying BDF $f$, whose value is unknown. Given as focal elements a finite number of intervals, $Y^{j}=\left[\underline{x}_{j}, \bar{x}_{j}\right]$, the pignistic transformation induced by a BBA $m_{X}\left(Y^{j}\right)$ is:

$$
\operatorname{Betf}(x)=\sum_{Y^{j}: x \in Y^{j}} \frac{m_{X}\left(Y^{j}\right)}{\left(\bar{x}_{j}-\underline{x}_{j}\right)\left(1-m_{X}(\varnothing)\right)}
$$

If we relax the assumption that the number of focal elements is finite, the pignistic probability induced by the BDF $f(\underline{x}, \bar{x})$ becomes:

$$
\operatorname{Betf}(x)=\lim _{\varepsilon \rightarrow 0} \int_{0}^{t} \int_{t+\varepsilon}^{R U L_{J}^{\max }} \frac{f(\underline{x}, \bar{x})}{\bar{x}-\underline{x}} d \underline{x} d \bar{x}
$$

As shown in [37], many BDFs $f(\underline{x}, \bar{x})$ can induce the same pignistic probability $\operatorname{Betf}(x)$ according to the transformation in eq. (22). The set of BDFs whose related pignistic pdfs equal Betf is called the set of isopignistic BDFs induced by Betf. Given two BDFs $f_{1}$ and $f_{2}$, we say that $f_{1}$ is more committed than $f_{2}$ if for all sets $Y^{j}$ it holds that $\operatorname{Bel}_{f_{1}}\left(Y^{j}\right)>\operatorname{Bel}_{f_{2}}\left(Y^{j}\right)$. In this work, we evoke the least commitment principle (never give more belief than needed to a subset of $\Omega_{R U L_{J}^{\text {test }}}$ ) to select the least committed (LC) isopignistic BDF $f_{R U L_{J}}^{G P R}(\underline{R U L}, \overline{R U L})$ induced by the pignistic pdf $\operatorname{Betf}_{G P R}\left(r u l_{J}\right)=p_{R U L_{J}^{t s t}}\left(r u l_{J} \mid \mathbf{D}_{\tau / z}^{+}\right)$. In Theorem 7.7 of [37] it is proven that the LC BDF $f_{R U L_{J}}^{G P R}(\underline{R U L}, \overline{R U L})$ of a unimodal pignistic pdf has focal sets which satisfy:

$$
\operatorname{Betf}_{G P R}(\underline{R U L})=\operatorname{Betf}_{G P R}(\overline{R U L})
$$


and has a belief density:

$$
f_{R U L_{J}}^{G P R}(\underline{R U L}, \overline{R U L})=\left[\overline{R U L}-\underline{R U L]} \frac{\mathrm{d} \operatorname{Betf} f_{G P R}(\underline{R U L})}{\mathrm{d} \underline{R U L}} \delta_{[\underline{R U L}, \phi(\underline{R U L})]} .\right.
$$

where $\phi(\underline{R U L})$ is a function of $\underline{R U L}$ that uniquely defines (since $\operatorname{Betf}(\overline{R U L})$ is a bell-shaped density) the value of $\overline{R U L}$ verifying the condition in eq. (23). Let $\mu=\operatorname{argmax}\left[\operatorname{Betf}_{G P R}\left(r u l_{J}\right)\right]$ be the mode of the unimodal pignistic pdf $B e t f_{G P R}$, then, the focal intervals $[\underline{R U L}, \phi(\underline{R U L})]$ of the LC BDF $f_{R U L_{J}}^{G P R}$ form a line in the half plane of all possible intervals which starts from point $(\mu, \mu)$ (see Figure 4, right).
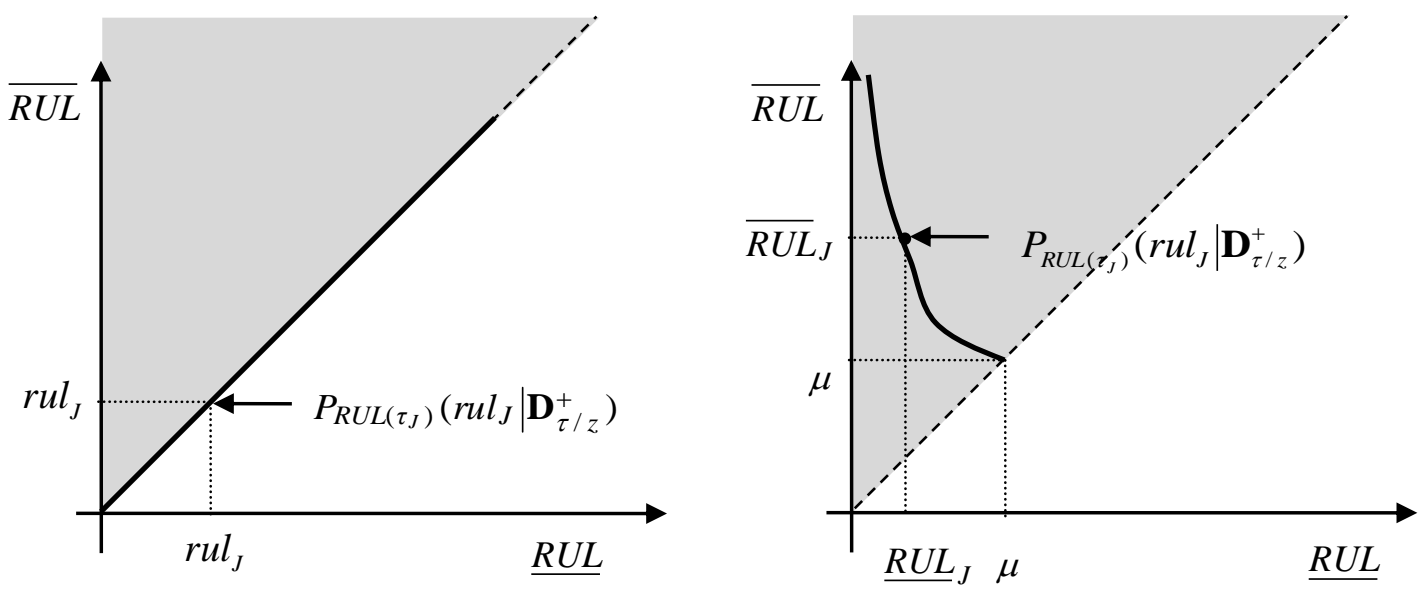

Figure 4: Graphical representation of the two possible transformations $f_{G P R 1}(\underline{R U L}, \overline{R U L})$ and $f_{G P R 2}(\underline{R U L}, \overline{R U L})$ of the RUL probability density function supplied by the GPR into a belief density function.

Once the RUL pdf predicted by the GPR approach is expressed in the form of the BDF function, $f_{R U L_{J}}^{G P R}(\underline{R U L}, \overline{R U L})$, it can be combined with the similarity-based prediction expressed by the BDF $f_{R U L_{J}}^{S B}(\underline{R U L}, \overline{R U L})$. To this aim, we have used a generalization of the Dempster's rule in the continuous frame of the real axis $\mathfrak{R}$, i.e., the conjunctive combination rule proposed by Smets in [37]. According to this rule, considering two BDFs, $f^{1}$ and $f^{2}$, a BDF $f_{1}\left(\left[\underline{x}_{1}, \bar{x}_{1}\right]\right) f_{2}\left(\left[\underline{x}_{2}, \bar{x}_{2}\right]\right)$ is assigned to the interval $[\underline{x}, \bar{x}]=\left[\underline{x}_{1}, \bar{x}_{1}\right] \cap\left[\underline{x}_{2}, \bar{x}_{2}\right]$ (which may also be empty). In our case, the aggregation of $f_{R U L_{J}}^{G P R}(\underline{R U L}, \overline{R U L})$ and $f_{R U L_{J}}^{S B}(\underline{R U L}, \overline{R U L})$ provides non-empty intervals in the following three cases:

1. intersection of the focal elements $\left[R U L_{j^{*}}^{r}, R U L_{j^{*}}^{r}\right]$ of $f_{R U L_{J}}^{S B}$ with the focal elements of $f_{R U L_{J}}^{G P R}$ only in the cases in which $\underline{R U L} \leq R U L_{j^{*}}^{r}$ and $\overline{R U L} \leq R U L_{j^{*}}^{r}$;

2. intersection of the focal element $\Omega_{R U L_{J}^{\text {est }}}$ of $f_{R U L_{J}}^{S B}$ with all the focal elements of $f_{R U L_{J}}^{G P R}$ only in the cases in which $\overline{R U L} \leq R U L_{J}^{\max }$;

3. intersection of the focal element $\Omega_{R U L_{J}^{\text {est }}}$ of $f_{R U L_{J}}^{S B}$ with all the focal elements of $f_{R U L_{J}}^{G P R}$ only in the cases in which $\underline{R U L} \leq R U L_{J}^{\max }$ and $\overline{R U L}>R U L_{J}^{\max }$. 
In case 1., we obtain as focal elements of the aggregated BDF the degenerate intervals $\left[R U L_{j^{*}}^{r}, R U L_{j^{*}}^{r}\right]$, $r=1, \ldots, R$. The mass of belief assigned to these focal elements is obtained by integrating the product $f_{R U L_{J}}^{S B}\left(R U L_{j^{*}}^{r}, R U L_{j^{*}}^{r}\right) f_{R U L_{J}}^{G P R}(\underline{R U L}, \overline{R U L})$ over all possible values $\underline{R U L}<R U L_{j^{*}}^{r}$ and $\overline{R U L}=<R U L_{j^{*}}^{r}$ :

$$
\begin{aligned}
& f_{R U L_{J}}^{S B}\left(R U L_{j^{*}}^{r}, R U L_{j^{*}}^{r}\right) \int_{0}^{R U L_{j^{*}}^{r}} \int_{R U L_{j^{*}}^{r}}^{\max } f_{R U L_{J}}^{G P R}(\underline{R U L}, \overline{R U L}) \mathrm{d} \underline{R U L} \mathrm{~d} \overline{R U L}= \\
& m_{R U L_{J}^{\text {test }}}\left(\left\{r \hat{u} l_{j^{*}}^{r}\right\}\right) \mathrm{Pl}_{R U L_{J}}^{G P R}\left(\left[R U L_{j^{*}}^{r}, R U L_{j^{*}}^{r}\right]\right) .
\end{aligned}
$$

In case 2., we obtain as focal elements of the aggregated BDF the intervals $[\underline{R U L}, \overline{R U L}]$ with $\overline{R U L}=\phi(\underline{R U L})$. The mass of belief assigned to these focal elements is proportional to:

$$
f_{R U L_{J}}^{S B}\left(0, R U L_{J}^{\max }\right) f_{R U L_{J}}^{G P R}(\underline{R U L}, \phi(\underline{R U L}))=m_{R U L_{J}^{t s t}}\left(\Omega_{R U L_{J}^{t e s t}}\right) f_{R U L_{J}}^{G P R}(\underline{R U L}, \phi(\underline{R U L})) .
$$

In case 3., we obtain as focal elements of the aggregated BDF the intervals $[\underline{R U L}, \overline{R U L}]$ with $\overline{R U L}=R U L_{J}^{\mathrm{max}}$ and the mass of belief assigned to these focal elements is the same of that assigned in case 2 . Thus, the combined BDFs $f_{R U L_{J}}^{C o m b}(\underline{R U L}, \overline{R U L})$ is

$$
\begin{aligned}
f_{R U L_{J}}^{C o m b}(\underline{R U L}, \overline{R U L})= & \frac{1}{K}\left[\sum_{r=1}^{R} m\left(\left\{r \hat{u} l_{j^{*}}^{r}\right\}\right) \operatorname{Pl}([\underline{R U L}, \overline{R U L}]) \cdot \delta_{\left(r \hat{u} l_{j^{*}}^{r}, r \hat{u} l_{j^{*}}^{r}\right.}(\underline{R U L}, \overline{R U L})\right. \\
& \left.+m\left(\Omega_{R U L_{J}^{t e s t}}\right) f(\underline{R U L}, \phi(\underline{R U L})) \cdot \delta_{\left(\underline{R U L}, \overline{R U L} L^{*}\right)}(\underline{R U L}, \overline{R U L})\right]
\end{aligned}
$$

where $K$ is a normalization constant that ensures that $f_{R U L_{J}}^{C o m b}(\underline{R U L}, \overline{R U L})$ integrates to 1 and where, for ease of notation, we have used $m=m_{R U L_{J}^{t_{J} \text { st }}}, f=f_{R U L_{J}}^{G P R}, P l=P l_{R U L_{J}}^{G P R}$ and $\overline{R U L^{*}}=\min \left(\phi(\underline{R U L}), R U L_{J}^{\max }\right)$.

Analogously to what done in Section 4 for the similarity-based approach, given the combined BDF $f_{R U L_{J}}^{C o m b}(\underline{R U L}, \overline{R U L})$, a credible left-bounded prediction interval for the value of the RUL is estimated by taking the interval $C_{J}^{C o m b}(\alpha)=\left[r u l_{J}^{\text {inf,Comb }}(\alpha),+\infty\right]$ to which $f_{R U L_{J}}^{C o m b}$ assigns the belief $\alpha$.

\section{Filter clogging prognostics - a case study}

In this Section, we consider the problem of predicting the RUL of filters used to clean the sea water entering the condenser of the BWR reactor of a Swedish nuclear power plant. During operations, filters undergo clogging and, once clogged, they can cumulate particles, seaweed, and mussels from the cooling water in the heat exchanger. Predictive maintenance can help achieving effective cleaning of the filters keeping maintenance costs reasonably low. 
From data collected in the field, we have available $N^{t s t}=8$ sequences of observations $\mathbf{z}_{1: n q}^{q}, q=1: N^{t s t}$ taken during the clogging process of $Q=8$ historical filters. Each observation $\mathbf{z}_{j}^{q}=\left[\Delta P_{j}^{q}, \dot{M}_{j}^{q}, T_{j}^{q}\right]$ contains the measurements of the pressure drop $\Delta P_{j}^{q}$, the flow across the filter $\dot{M}_{j}^{q}$, and the sea water temperature $T_{j}^{q}$ collected at time $\tau_{j}^{q}$ during the clogging process of the $q$-th filter. To apply degradation-based prognostics, it is necessary to derive from the observations $\mathbf{z}_{j}^{q}$ an indication of the degradation state of the filter, i.e. its clogging, at time $\tau_{j}$. An increasing number of articles can be found in the literature concerning the study of filter clogging by solid aerosols [38] and liquid aerosols [13]. These articles describe the results achieved in controlled environment where all degradation quantities, indicators of degradation and stressors are automatically measured and recorded. Although this is not the situation encountered in this industrial case study, it has been well established that the clogging of a filter medium leads to an increase in pressure drop over the filter as long as the filtration velocity, and thus the flow, is kept constant. It is also known that the pressure drop is proportional to the square of the filtration velocity. Given these results, we consider an indicator of filter $q$ degradation at time $\tau_{j}$ the ratio [26]:

$$
z_{\delta, j}^{q}=\frac{\Delta P_{j}^{q}}{\left(\dot{M}_{j}^{q}\right)^{2}}
$$

Also, due to the absence of physical knowledge about the failure threshold, this has been arbitrarily set to the value $\delta^{\text {th }}=175$ by looking at the available data and the corresponding values derived (eq. (28)) for the degradation indicator observations. Figure 5 shows the sequences of degradation observations $z_{\delta, 1: n q}^{q}$, $q=1: N^{\text {tst }}$ collected in the field during the clogging process of the 8 filters. It can be observed that the clogging process is affected by large uncertainties, which are due to the very variable conditions of the sea water; in this situation, the challenge is to provide sufficiently narrow confidence intervals for the value of the RUL predictions.

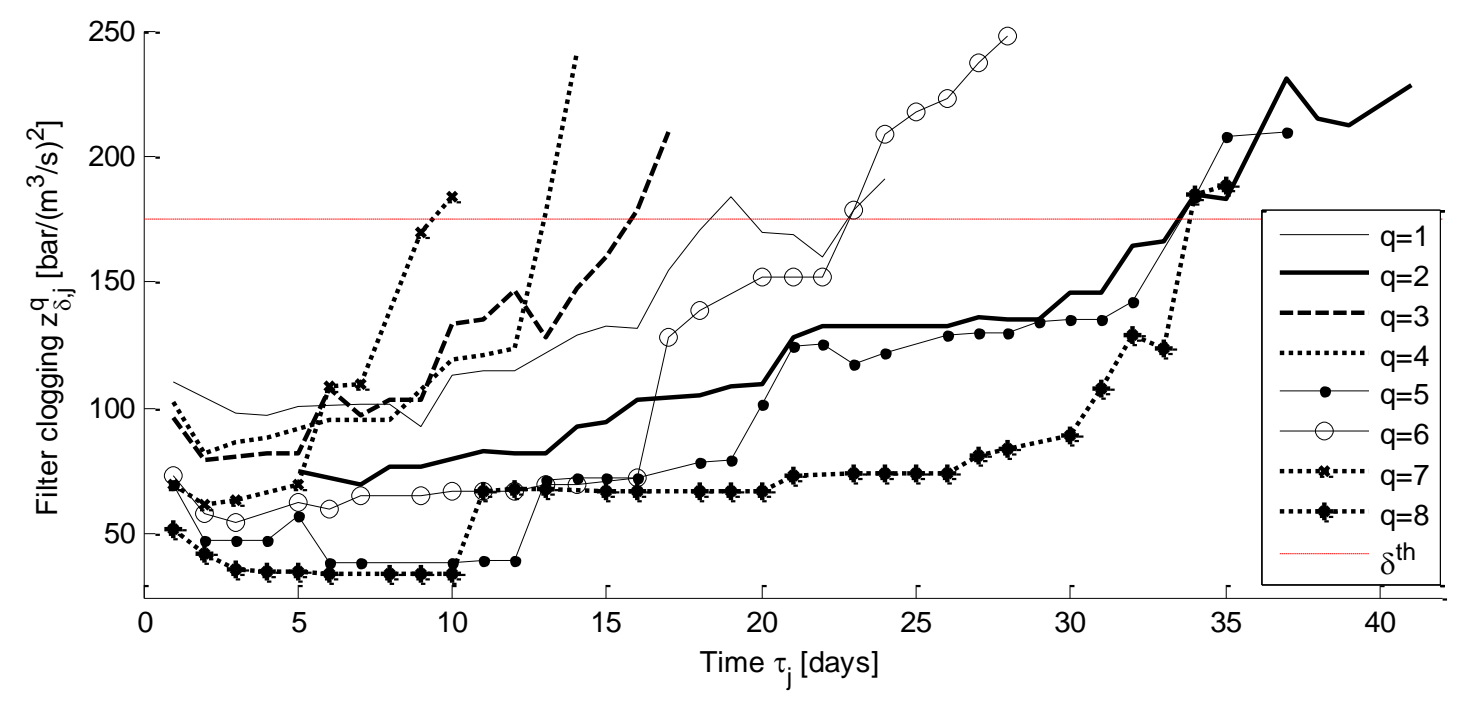

Figure 5: time evolution of the degradation $\mathbf{z}_{1: n q}^{q}$ in the available $q=1: 8$ trajectories

\subsection{Results}


The two prognostic approaches proposed in Sections 3 and 4 and the combined ensemble strategy proposed in Section 5 are applied for the prediction of the filter RUL at all time instants of each trajectory. In practice, $\mathrm{R}=7$ trajectories are used to train the prognostic models and the remaining trajectory to verify its performance. This leave-one-out procedure $[7,29]$ has been repeated 8 times, using each time a different test trajectory.

With respect to the GPR approach, the priors of the mean and covariance function of the GP used to model the clogging process are set, according to [8], as:

$$
\begin{aligned}
& m_{\Delta}(\tau)=\sum_{i=0}^{3} a_{i} \tau^{i} \\
& k_{1}\left(\tau, \tau^{\prime}\right)=b_{1} \exp \left(\frac{-\left(\tau-\tau^{\prime}\right)^{2}}{b_{2}}\right) \\
& k_{2}\left(\tau, \tau^{\prime}\right)=c_{1} \tau \cdot \tau^{\prime}+c_{2}+c_{3} \sin ^{-1}\left(\frac{c_{4} \tau \tau^{\prime}}{\sqrt{\left(1+c_{4} \tau^{2}\right)\left(1+c_{4} \tau^{\prime 2}\right)}}\right)+
\end{aligned}
$$

where $a_{0: 3}, b_{1: 2}$, and $c_{1: 4}$ are the hyper-parameters optimized by maximizing the marginal likelihood of the dataset $\mathbf{D}_{\tau / z}^{\text {train }}$ derived from the training trajectories.

With respect to the similarity-based approach, we used parameters $\lambda=0.05$ and $\gamma=0.95$ according to the results obtained in [3].

Figures 6 and 7 show the RUL prediction and the left bound of the prediction interval with belief $\alpha=0.9$ supplied by the GPR and the similarity-based approaches. Due to the large uncertainty of the process, the accuracy in the RUL prediction obtained by the two approaches is low and the confidence intervals are large. The GPR approach provides in general narrower prediction intervals than the similarity-based approach, which tends to provide a lower bound of the RUL prediction interval often equal to zero. As pointed out by [3], this does not mean that the evidence of very early failure is high (as demonstrated by the fact that the predicted RUL can be far from zero), but only that the evidence drawn from the reference trajectories is not sufficient to assert with the desired belief $\alpha=0.9$ that the RUL value is larger than 0 . In other words, the prediction $r u l_{J}^{\mathrm{inf}, S B}=0$ is a statement of ignorance about the value of $R U L_{J}^{\text {test }}$. Such large prediction intervals cannot be used by an operator who is asked to make a choice about the best time for undertaking a maintenance action. However, the large intervals predicted by the similarity-based approach can provide a correct indication that the information conveyed by the training trajectories is not relevant for a specific test trajectory, e.g., because they are too dissimilar. This can be seen, in particular, for trajectories $q=4$ and $q=7$ where the GPR approach provides narrower prediction intervals, but such intervals do not include the true RUL value. Notice also that, for these two trajectories, the prediction $r \hat{u} l_{J}^{S B}$ is more accurate than $r \hat{u} l_{J}^{G P R}$

Figure 8 (dots) shows the RUL prediction $r \hat{u} l_{J}^{C o m b}$ obtained by averaging the GPR and the similarity-based approaches, and the lower bounds of the prediction intervals for $\alpha=0.9$ obtained by the ensemble combination approach presented in Section 5. We notice that, since the information conveyed by the pdf $p_{R U L_{J}^{\text {lest }}}\left(r u l_{J} \mid \mathbf{D}_{\tau / z}^{+}\right)$is relaxed through the LC transformation performed in the ensemble combination, the resulting $\mathrm{BDF} f_{R U L_{J}}^{C o m b}(\underline{R U L}, \overline{R U L})$ is influenced by the information conveyed by both the GPR and the 
similarity-based BBAs. This can be observed for trajectories $q=4$ and $q=7$, where $f_{R U L_{J}}^{C o m b}(\underline{R U L}, \overline{R U L})$ provides larger confidence bounds than the GPR approach, thus appearing to be more robust, since its prediction intervals include the true RUL values and, at the same time, are narrower than those provided by the similarity-based approach.
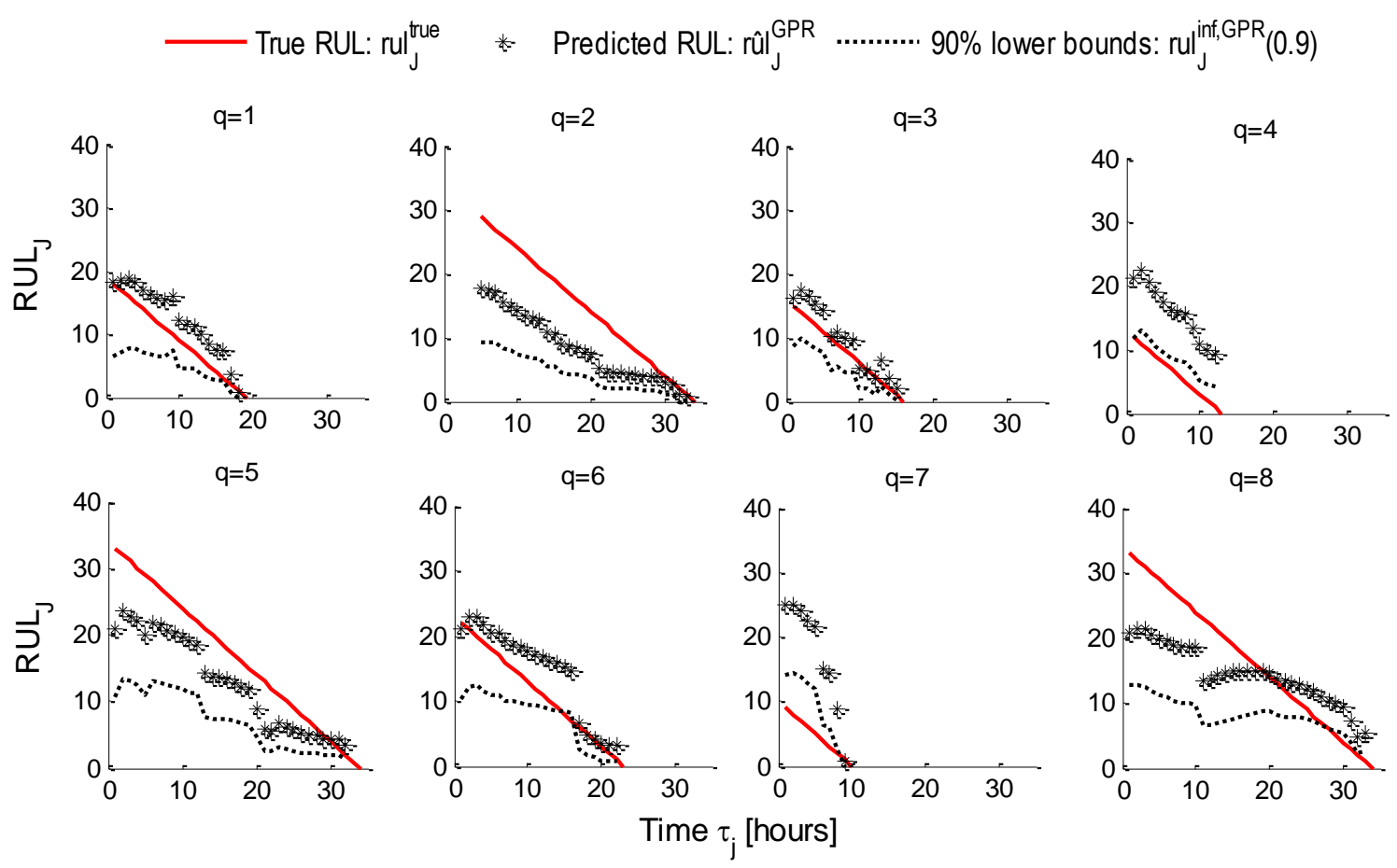

Figure 6: Predictions rûl ${ }_{J}^{G P R}$ (asterisks) and $90 \%$ left bounded prediction interval rul $l_{J}^{\mathrm{inf}, G P R}(0.9)$ (dots) provided by the GPR approach.

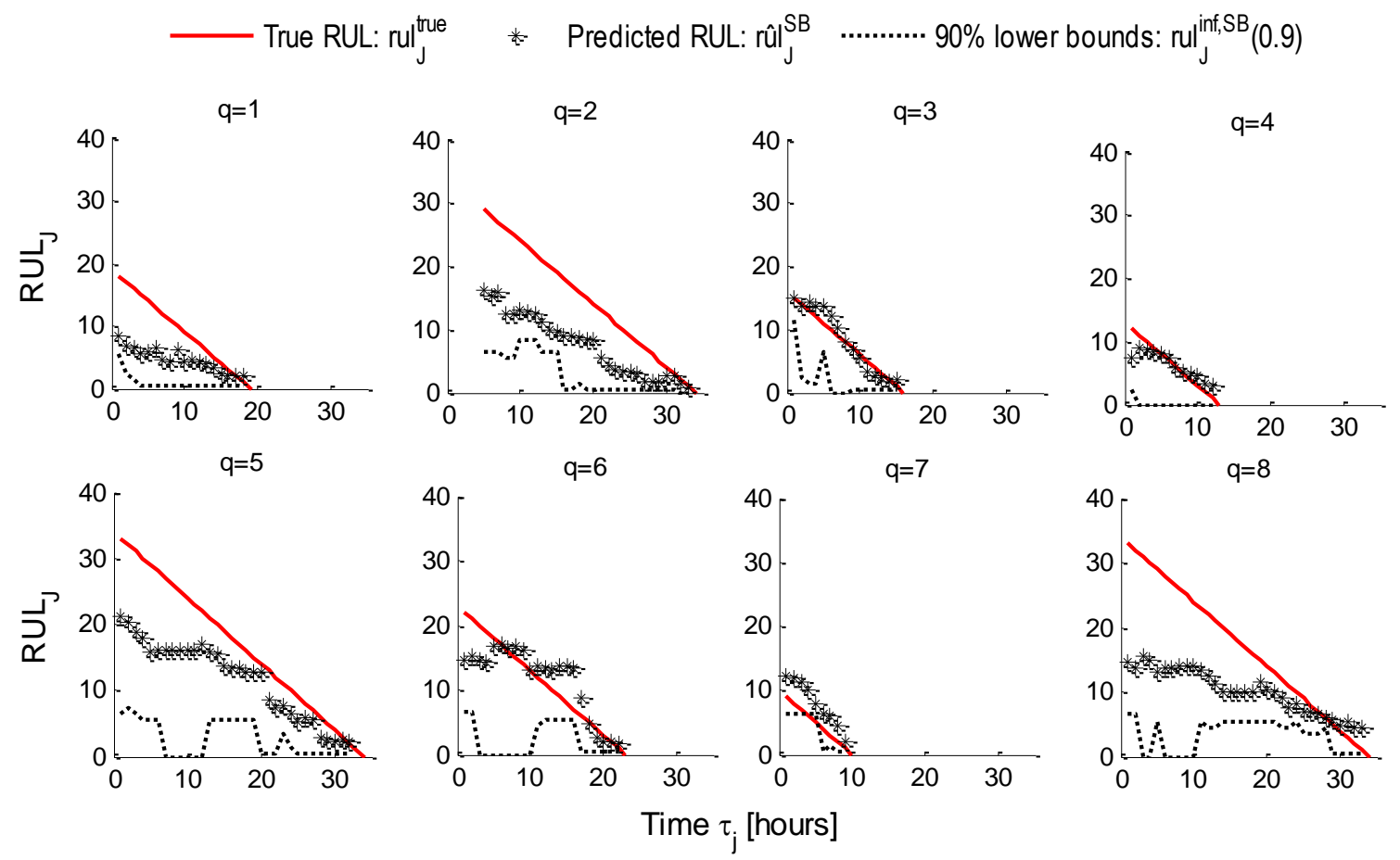

Figure 7: Prediction rull $\left.\right|_{J} ^{S B}$ (asterisks) and $90 \%$ left bounded prediction interval rul $l_{J}^{\mathrm{inf}, S B}(0.9)$ (dots) provided by the similaritybased approach. 


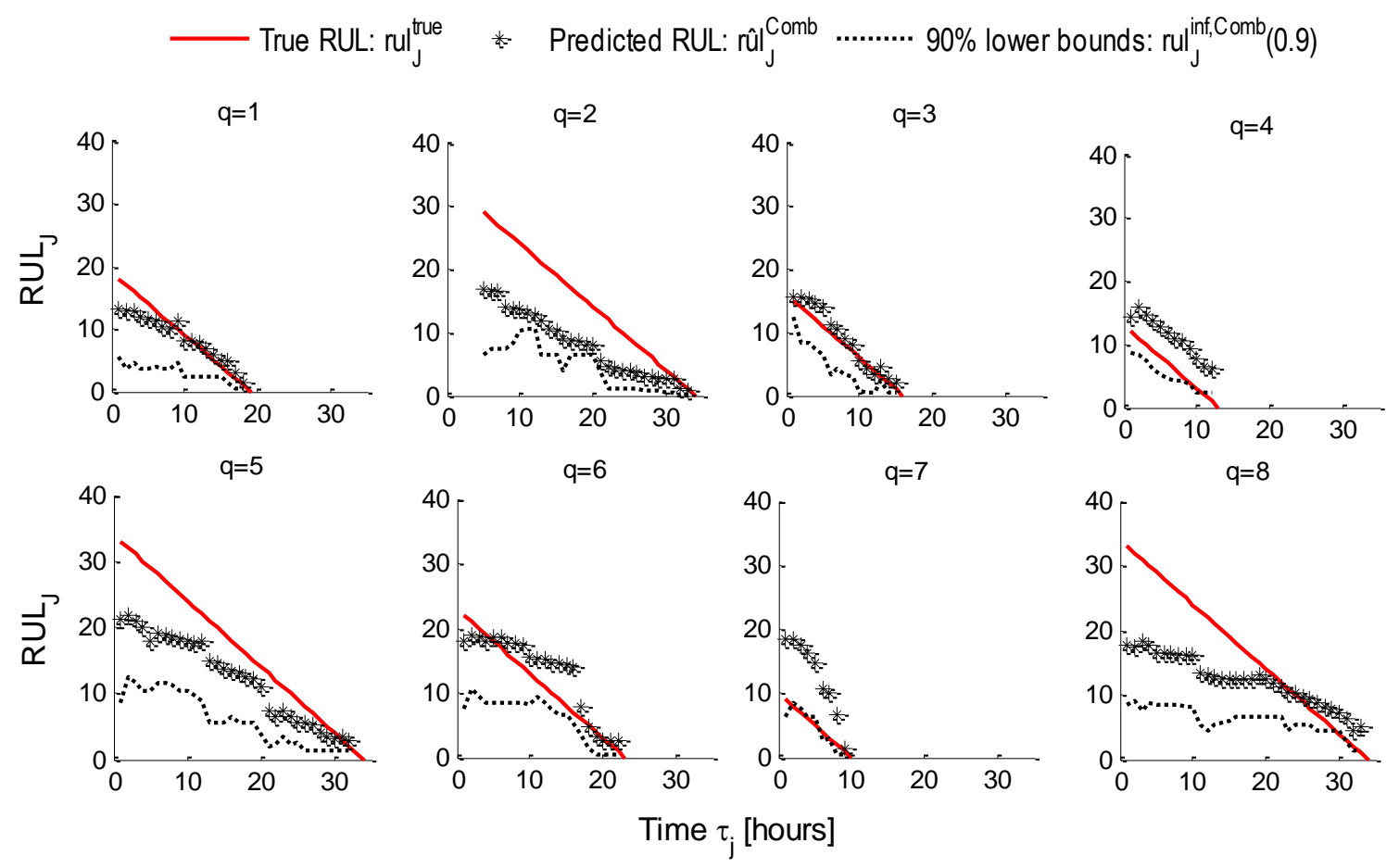

Figure 8: Prediction rûl ${ }_{J}^{\text {Comb }}$ (asterisks) and $90 \%$ left bounded prediction interval rul $\mathrm{inf}_{J}^{\mathrm{in} C o m b}(0.9)$ (dots) provided by the ensemble combination.

To further evaluate the different approaches, they are applied to each trajectory $q=1, \ldots, 8$ in correspondence of the life fraction $\beta_{1}=0.3, \beta_{2}=0.6, \beta_{3}=0.8$ and $\beta_{4}=0.95$, i.e., at time step $\tau_{J(\beta)}^{q}=\beta \cdot \tau_{F}^{q}$, with $\tau_{F}^{q}$ indicating the failure time of filter $q$, and the obtained predictions $r \hat{u} l_{J(\beta)}^{q}$ and $C I_{J(\beta)}^{q}(0.9)=\left[r \hat{u} l_{J(\beta)}^{\text {inf, } q},+\infty\right)$ are analyzed. In particular, the following performance indicators are considered:

- the square root of the Mean Square Error (RMSE), i.e., the average value over all the 8 clogging trajectories $q=1: 8$ of the square error $\left(r \hat{u} l_{J(\beta)}^{q}-r u l_{J(\beta)}^{q, \text { true }}\right)^{2}$ made in predicting the true RUL of the test equipment. The MSE measures the accuracy of the prediction and is desired to be as small as possible.

- the amplitude $\left(\mathrm{MA}_{0.9}\right)$ of the interval $\left[r \hat{u} l_{J(\beta)}^{\mathrm{inf}, q}, R U L_{J(\beta)}^{\max }\right]$, where $R U L_{J(\beta)}^{\max }=\tau_{F}^{\max }-\beta \tau_{F}^{q}$ indicates the maximum RUL of filters that have been already operating for a time span of $\beta \tau_{F}^{q}$, averaged over all clogging trajectories. The value $R U L_{J(\beta)}^{\max }$ has been considered instead of $+\infty$, in order to allow a quantification of the interval. For having high precision and avoiding unnecessarily early maintenance interventions, we wish to have the value of $\mathrm{MA}_{0.9}$ as small as possible.

A third indicator, the coverage $\operatorname{Cov}_{0.9}$, is evaluated over all the predictions performed at each time instant $\tau_{J}^{q}$ along the clogging trajectories by considering the percentage of times the condition $r u l_{J(\beta)}^{q, t r u e} \in C I_{J(\beta)}^{q}(0.9)$ is verified. This indicator measures the reliability of the left bounded prediction interval (it is required to be larger than $\alpha=0.9$ ).

Figure 9 shows the value of these indicators obtained for the GPR and similarity-based approaches, and their ensemble combination. An horizontal (dotted) line indicates the target value $\alpha=0.9$ for the coverage (upper, left). 


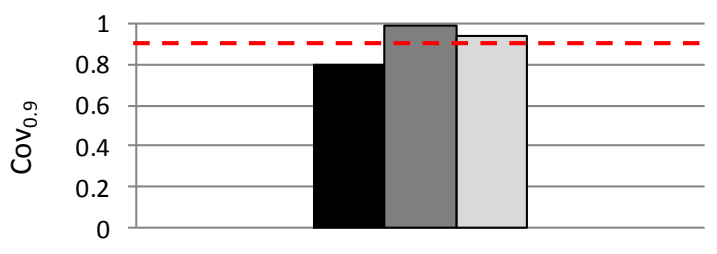

life fraction $\beta$

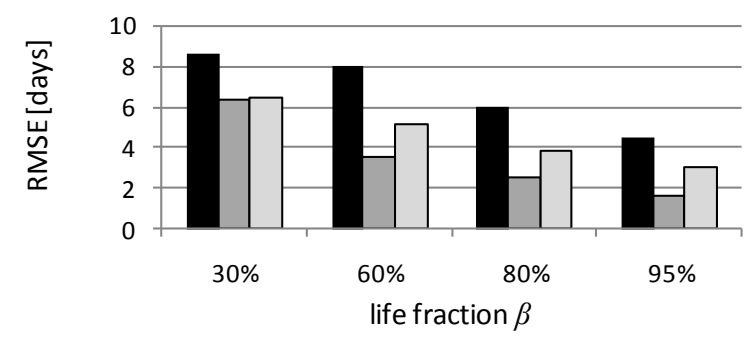

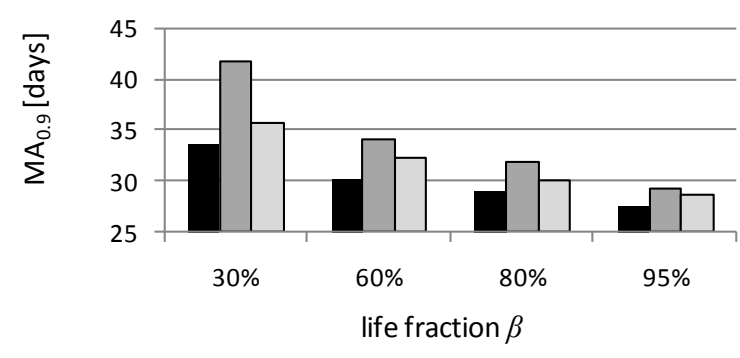

घPR $\square$ Similarity $\square$ Combined $\square$ Average RUL

Figure 9: Comparison of the performance indicators Covo.9 (upper, left), MA 0.9 (upper, right) and RMSE (bottom, left) for the three approaches. The horizontal (dotted) line in the Covo.9 graph (upper, left) indicates the target coverage value $\alpha=0.9$.

Figure 9 shows that, as expected, the error and the amplitude of the prediction interval decrease with the equipment life. The comparison of the average performances of the two individual approaches shows that the SB approach is more satisfactory since it provides more accurate predictions (lower RMSE) and can assure the desired coverage level. This is due to the remarkable errors made by the GPR approach in the predictions of trajectories 4 and 7 RULs. The drawback of the SB approach is that it is characterized by very large prediction intervals. The ensemble combination of the two methods, instead, allows obtaining smaller prediction intervals than the similarity-based approach, and, at the same time, it ensures the desired coverage level. Thus, if we consider the point of view of an operator who is asked to make a choice about the best time for undertaking a maintenance action according to a desired risk tolerance (90\% confidence that the failure is after the maintenance intervention time), we can conclude that:

- the $90 \%$ left bounded prediction intervals provided by the GPR approach do not allow to meet the desired risk tolerance criterion;

- the $90 \%$ left bounded prediction intervals provided by the SB approach will cause too early maintenance interventions;

- the $90 \%$ left bounded prediction intervals provided by the ensemble combination should be preferred since they allow to meet the desired risk tolerance criterion, and, at the same time, to avoid the unnecessary anticipation of the maintenance intervention typical of the SB approach.

On the other side, if we consider the RUL point estimate, we notice that the average accuracy of the ensemble combination is slightly less satisfactory than that of the similarity-based approach. This depends on the strategy used for the aggregation of $r \hat{u} l_{J}^{G P R}$ and $r \hat{u} l_{J}^{S B}$ which is based on a simple average and which causes very unsatisfactory predictions for trajectories 4 and 7 characterized by high errors of $r \hat{u} l_{J}^{G P R}$. In this respect, we conjecture that other strategies for the aggregation of $r \hat{u} l_{J}^{G P R}$ and $r \hat{u} l_{J}^{S B}$, accounting, e.g., for the 
historical performance of the two methods, have the potential of improving the accuracy of the prediction $r u \hat{u} l_{J}^{\text {Comb }}$.

\section{Conclusions}

In this work, we have considered the problem of predicting the RUL of degrading equipment and providing a measure of its uncertainty, based on sequences of observations collected during the degradation trajectories of a set of similar equipments which have failed in the past. Two different prognostic approaches have been considered: a degradation-based approach resorting to Gaussian process regression to model the evolution of the equipment degradation and predict the probability distribution of the equipment RUL, and a direct RUL prediction approach which exploits similarity-based regression and belief function theory for inferring a basic belief assignment for the value of the test equipment RUL.

The application of the two methods to real data concerning the clogging of filters used in a BWR condenser has shown that the prediction intervals provided by the SBR approach have good coverage, but are extremely large, whereas those provided by the GPR are narrower but do not achieve the desired coverage.

A third approach has, then, been proposed, which is based on the ensemble aggregation of the outcomes of these two complementary methods. The main difficulty in performing such aggregation has been the necessity of combining two different representations of uncertainty, based, respectively, on probabilistic and evidential reasoning. By resorting to the belief function theory on continuous variables, it has been possible to translate both representations of the uncertainty variable $R U L$ within the same framework and, subsequently, aggregate them using Dempster's rule.

The aggregation of the predictions provided by the GPR and the SBR approaches represents a good compromise since it allows to reach the desired coverage, contrarily to the GPR predictions, keeping the prediction intervals narrower than those provided by the SBR approach alone.

Notice that the aggregation method proposed in this paper with reference to the aggregation of two specific prognostic methods can be applied to any situation requiring the combination of multiple uncertain predictions represented in the different frameworks of probabilistic and evidential reasoning.

Concerning the accuracy of the combined RUL prediction, future research should consider aggregation strategies other than the simple average, e.g., performance-based aggregation strategies, which, by accounting for the prediction error made by each approach on historical validation data, have the potential of improving the accuracy of the aggregated prediction.

Furthermore, in a situation where one is very confident about the accuracy and reliability of the available RUL pdf, isopignistic transformations other than that based on the least commitment principle, should be considered, since they have the potential of reducing the amplitude of the prediction intervals.

\section{Acknowledgments}


The work of Francesca Mangili has been supported by a PhD grant of the Institutt For Energiteknikk (IFE), OECD Halden Reactor Project. The participation of Enrico Zio to this research is partially supported by the China NSFC under grant number 71231001.

\section{References}

[1] Baraldi, P., Cammi, A., Mangili, F., Zio, E., 2010. Local Fusion of an Ensemble of Models for the Reconstruction of Faulty Signals - IEEE Transactions on Nuclear Science, vol. 57, pp. 793- 806.

[2] Baraldi, P., Compare, M., Zio, E. 2013. Maintenance policy performance assessment in presence of imprecision based on Dempster-Shafer Theory of Evidence, Information Sciences, Vol 245, pp. 112-131

[3] Baraldi, P., Di Maio, F., Mangili, F., Zio, E., 2013. A belief function theory method for prognostics in clogging filters. Chemical Engineering Transactions, 33, pp. 847-852.

[4] Baraldi, P., Mangili, F., Zio, E., 2013b. Investigation of uncertainty treatment capability of model-based and data-driven prognostic methods using simulated data. Reliability Engineering and System Safety, Vol 112C, pp. 94-108.

[5] Baraldi, P., Zio, E., 2008. A Combined Monte Carlo and possibilistic approach to uncertainty propagation in event tree analysis, Risk Analysis, 28 (5), pp. 1309-1325

[6] Baraldi, P., Zio, E., 2010 A comparison between probabilistic and Dempster-Shafer Theory approaches to Model Uncertainty Analysis in the Performance Assessment of Radioactive Waste Repositories". Risk Analysis, Vol. 30 (7), pp. 1139-1156, 2010.

[7] Baraldi, P., Zio, E., Gola, G., Roverso, D., Hoffmann, M., 2011. Two novel procedures for aggregating randomized model ensemble outcomes for robust signal reconstruction in nuclear power plants monitoring systems, Annals of Nuclear Energy, Vol. 38 (2-3), pp. 212-220.

[8] Baraldi, P., Mangili, F., Zio, E., 2013. A prognostics approach to nuclear component degradation modeling based on Gaussian Process Regression. Submitted to Progress in nuclear energy.

[9] Baraldi, P. Di Maio, F., Zio, E. ,Sauco, S. Droguett, E. Magno C., 2012. Ensemble of Neural Networks for Predicting Scale Deposition in Oil Well Plants Equipments, PSAM 11 \& ESREL 2012 Conference, Joint Probabilistic Safety Assessment and Management Conference and European Safety and Reliability Conference, Helsinki, Finlandia, 25-29 June 2012.

[10] Benkedjouh, T., Medjaher, K., Zerhouni, N., Rechak, S., 2013. Health assessment and life prediction of cutting tools based on support vector regression. Journal of Intelligent Manufacturing, article published online.

[11] Bonissone, P., Xue, F., Subbu, R., 2008. Fast Meta-models for Local Fusion of Multiple Predictive Models. Applied Soft Computing Journal 11(2), 1529-1539.

[12] Clemen, R. T. andWinkler, R. L. (2007) Aggregating probability distributions. In Advances in Decision Analysis: from Foundations to Applications (eds E.Ward, R. F. Miles and D. vonWinterfeldt), pp. 154-176. Cambridge: Cambridge University Press.

[13] Contal, P., Simao, J., Thomas, D., Frising, T., Callé, S., Appert-Collin, J.C., Bémer, D., 2004. Clogging of fibre filters by submicron droplets. Phenomena and influence of operating conditions. Aerosol Science, Vol. 35, pp. 263-278.

[14] Dempster, A.P., 1976. Upper and lower probabilities induced by a multivariate mapping, Annals of Mathematical Statistics AMS-38 325-339.

[15] Goebel, K., Saha, B., Saxena, A., 2008. A Comparison of Three Data-Driven Techniques for Prognostics, proc. of the 62nd Meeting of the Society For Machinery Failure Prevention Technology (MFPT), May 6-8, Virginia Beach, Virginia.

[16] Gorjian, N., Ma, L., Mittinty, M., Yarlagadda, P., Sun, Y., 2009. Review on Degradation Models in Reliability Analysis, Proceedings of the 4th World Congress on Engineering Asset Management, 28-30 Sept, Athens.

[17] Helton, J. C., 2004. Alternative representations of epistemic uncertainty. Special Issue of Reliability Engineering and System Safety, 85,1-10

[18] Lee, J., Ni, J., Djurdjanovic, D., Qiu, H., Liao, H., 2006. Intelligent prognostics tools and e-maintenance. Computers in Industry, 57(6), 476-489

[19] Liu, R., Ma, L., Kang, R., Wang, N., 2011. The modeling method on failure prognostics uncertainties in maintenance policy decision process. Proc 9th Int Conf on Reliab, Maint and Saf (ICRMS), Jun 12-15; Guiyang, China.

[20] MacKay, D.J.C., 1998. Introduction to Gaussian processes. In:Bishop CM (ed) Neural networks and machine learning, vol 168. NATO ASI Series, Springer, Berlin, pp 133-165

[21] Mann, R., Freeman, R., Osborne, M., Garnett, R., Armstrong, C., Meade, J., Biro, D., Guilford, T., Roberts, S., 2011. Objectively identifying landmark use and predicting flight trajectories of the homing pigeon using Gaussian processes, Journal of the Royal Society Interface, Vol. 8, pp. 210-219.

[22] McCall, JJ, Maintenance policies for stochastically failing equipment: a survey, Management Science, 11 (1965), pp. 493524

[23] MIL-STD 721C. 1981. Definitions of terms for reliability and maintainability. United States-department of defense

[24] Mohanty, S., Chattopadhyay, A., Peralta, P., Das, S., 2011. Bayesian Statistic Based Multivariate Gaussian Process Approach for Offline/Online Fatigue Crack Growth Prediction. Experimental Mechanics, Vol. 51, pp. 833-843.

[25] Mosallam, A,, Medjaher, K., Zerhouni, N., 2014. Data-driven prognostic method based on Bayesian approaches for direct remaining useful life prediction, J Intell Manuf, Published online

[26] Nystad, B.H., 2009. Condition-Based Maintenance (CBM) - filter clogging at OKG 1, a case study, HWR-961, OECD Halden Reactor Project. 
[27] Peel, L., 2008. Data Driven Prognostics using a Kalman Filter Ensemble of Neural Network Models, International Conference on Prognostics and Health Management.

[28] Petit-Renaud, S., Denoeux, T., 2004. Nonparametric regression analysis of uncertain and imprecise data using belief functions. International Journal of Approximate Reasoing, Vol 35, pp.1-28.

[29] Polikar, R., 2006. Ensemble Based Systems in Decision Making, IEEE Circuits and Systems Magazine, Vol. 6(3), pp. 2145.

[30] Polikar, R., 2007. Bootstrap Inspired Techniques in Computational Intelligence, IEEE Signal Processing Magazine, Vol. 24(4), pp. 56-72.

[31] Rasmussen, C., Williams, C., 2006. Gaussian processes for machine learning. MIT Press, Cambridge, MA.

[32] Ristic, B., Smets, P., 2004. Belief function theory on the continuous space with an application to model based classification. In: IPMU (Ed.), Information Processing and Management of Uncertainty, 2004, pp. 1119-1126

[33] Ronald R. Yager, Vladik Kreinovich, Decision making under interval probabilities, International Journal of Approximate Reasoning, Volume 22, Issue 3, December 1999, Pages 195-215.

[34] Schwabacher, M., Goebel, K., 2007. A Survey of Artificial Intelligence for Prognostics. Association for the Advancement of Artificial Intelligence Fall Symposium, 9-11 Nov, Arlington (VA).

[35] Shafer, G., 1976. A mathematical theory of evidence, Princeton University Press. Princeton, NJ.

[36] Smets, P., 1998. The transferable belief model for quantified belief representation, in: D.M. Gabbay, P. Smets (Eds.), Handbook of Defeasible Reasoning and Uncertainty Management Systems, vol. 1, Kluwer Academic Publishers, Dordrecht, pp. 267-301.

[37] Smets, P., 2005. Belief functions on real numbers. International Journal of Approximate Reasoning, Vol 40 (3), pp. 181223.

[38] Song, C.B., Park, H.S., Lee, K.W., 2006. Experimental study of clogging with monodisperse PSL particles. Power Technology, Vol. 163, pp. 152-159.

[39] Tang, L., Kacprzynski, G.J., Goebel, K., 2009. Vachtsevanos G. Methodologies for Uncertainty Management in Prognostics. Proc IEEE Aerosp Conf, Mar 7-14; Big Sky, MT.

[40] Tipping, M.E., 2001. Sparse Bayesian Learning and the Relevance Vector Machine. Journal of Machine Learning Research, 1:211-244.

[41] Yager R., 1987 On the Dempster-Shafer framework and new combination rules. Information Sciences, 1987; 41:93-137. [34A] Yan, J., Koc, M., \& Lee, J. (2004). A prognostic algorithm for machine performance assessment and its application. Production Planning and Control, 76, 796-801

[42] K. Yamada, A new combination of evidence based on compromise, Fuzzy Sets and Systems 159 (2008) 1689-1708.

[43] Vachtsevanos, G., Wang, P., 2001. Fault prognosis using dynamic wavelet neural networks. Proceedings of IEEE Systems Readiness Technology Conference (AUTEST), pp 857-870

[44] Wang, W., Carr, M., Xu, W., Kobbacy KAH, A model for residual life prediction based on Brownian motion with an adaptive drift, Microelectronics Reliability, 51 (2011), pp. 285-293

[45] Wang, T., Yu, J., Siegel, D., Lee, J., 2008. A Similarity-Based Prognostics Approach for Remaining Useful Life Estimation of Engineered Systems. International Conference on Prognostics and Health Management, 2008, 6-9 Oct., Denver (CO).

[46] R.R. Yager, 2011. On the fusion of imprecise uncertainty measures using belief structures, Information Sciences, Vol 181 (15), pp. 3199-3209.

[47] Zhao W., T. Tao, ZhuoShu D., E. Zio, "A dynamic particle filter-support vector regression method for reliability prediction”, Reliability Engineering \& System Safety, Volume 119, November 2013, Pages 109-116,

[48] Zhao Z., Fu-li Wang, Ming-xing Jia, Shu Wang, Predictive maintenance policy based on process data, Chemometrics and Intelligent Laboratory Systems, Volume 103, Issue 2, 15 October 2010, Pages 137-143

[49] Zio, E., Compare, M., 2010. Evaluating maintenance policies by quantitative modeling and analysis. Reliability Engineering System Safety, Vol. 109, pp. 53-65.

[50]Zio, E., Di Maio, F., 2010. A data-driven fuzzy approach for predicting the remaining useful life in dynamic failure scenarios of a nuclear system. Reliability Engineering \& System Safety, Vol. 95(1), pp. 49-57. 